Math. Model. Nat. Phenom.

Vol. 6, No. 1, 2011, pp. 87-118

DOI: $10.1051 / \mathrm{mmnp} / 20116105$

\title{
Interaction of Turing and Hopf Modes in the Superdiffusive Brusselator Model Near a Codimension Two Bifurcation Point
}

\author{
J.C. Tzou, A. Bayliss, B.J. Matkowsky * and V.A. Volpert \\ Department of Engineering Sciences and Applied Mathematics \\ Northwestern University, Evanston IL 60208-3125 USA
}

This paper is dedicated to the memory of our colleague and friend Alexander (Sasha) Golovin

\begin{abstract}
Spatiotemporal patterns near a codimension-2 Turing-Hopf point of the one-dimensional superdiffusive Brusselator model are analyzed. The superdiffusive Brusselator model differs from its regular counterpart in that the Laplacian operator of the regular model is replaced by $\partial^{\alpha} / \partial|\xi|^{\alpha}$, $1<\alpha<2$, an integro-differential operator that reflects the nonlocal behavior of superdiffusion. The order of the operator, $\alpha$, is a measure of the rate of superdiffusion, which, in general, can be different for each of the two components. A weakly nonlinear analysis is used to derive two coupled amplitude equations describing the slow time evolution of the Turing and Hopf modes. We seek special solutions of the amplitude equations, namely a pure Turing solution, a pure Hopf solution, and a mixed mode solution, and analyze their stability to long-wave perturbations. We find that the stability criteria of all three solutions depend greatly on the rates of superdiffusion of the two components. In addition, the stability properties of the solutions to the anomalous diffusion model are different from those of the regular diffusion model. Numerical computations in a large spatial domain, using Fourier spectral methods in space and second order Runge-Kutta in time are used to confirm the analysis and also to find solutions not predicted by the weakly nonlinear analysis, in the fully nonlinear regime. Specifically, we find a large number of steady state patterns consisting of a localized region or regions of stationary stripes in a background of time periodic cellular motion, as well as patterns with a localized region or regions of time periodic cells in a background of stationary stripes. Each such pattern lies on a branch of such solutions, is stable and
\end{abstract}

\footnotetext{
${ }^{*}$ Corresponding author. E-mail: b-matkowsky@ northwestern.edu
} 
corresponds to a different initial condition. The patterns correspond to the phenomenon of pinning of the front between the stripes and the time periodic cellular motion. While in some cases it is difficult to isolate the effect of the diffusion exponents, we find characteristics in the spatiotemporal patterns for anomalous diffusion that we have not found for regular (Fickian) diffusion.

Key words: Brusselator model, superdiffusion, Turing pattern, Hopf bifurcation, codimension-2 bifurcation, weakly nonlinear analysis, amplitude equations, spatiotemporal patterns

AMS subject classification: 35B36, 35K57, 35Q99, 37G99

\section{Introduction}

Studies of anomalous diffusion have recently been appearing in the literature as more processes have been observed to exhibit behavior that cannot be described by regular (Fickian) diffusion. These processes can often be described by models with subdiffusion or superdiffusion, where, under a random walk description, the mean square displacement of a particle scales as $\left\langle x^{2}(t)\right\rangle \sim t^{\gamma}$, with $0<\gamma<1$ for subdiffusion, and $1<\gamma<2$ for superdiffusion, rather than linearly in time. Subdiffusion has been observed in many applications, including charge carrier transport in amorphous semiconductors, and nuclear magnetic resonance diffusometry in percolative and porous systems, while superdiffusion has been observed in e.g., transport in heterogeneous rocks, quantum optics, and single molecule spectroscopy [10]. We consider an especially interesting case of superdiffusion, Lévy flights, which is characterized by a jump length distribution having infinite moments. On the macroscopic scale, Lévy flights are described by a diffusion equation where the second order spatial derivative is replaced by a fractional derivative $\partial^{\alpha} / \partial|\xi|^{\alpha}, 1<\alpha<2$, defined as a non-local integro-differential operator [10]. Previous works on reaction-superdiffusion equations have derived and studied amplitude equations near a Hopf [11] or Turing [8] bifurcation point. In this paper, we investigate the effects of superdiffusion on the interactions between Hopf and Turing instabilities of the Brusselator model by deriving amplitude equations and studying instabilities of their solutions to long-wave perturbations, thus leading to the identification of the parameter values at which new solutions may bifurcate. Similar studies near a codimension-two Turing-Hopf point (C2THP) of the regular Brusselator model have been done in [7], [15]. We also discuss the results of numerical computations in both the weakly and fully nonlinear regimes. In this paper, we consider both the regular and superdiffusive one-dimensional Brusselator model and identify characteristics of spatiotemporal patterns obtained near the C2THP that are unique to each. In the anomalous case, we consider cases of equal diffusion exponents, unequal but close diffusion exponents, and where one diffusion is regular while the other is anomalous. 


\section{The model, the basic solution, and its linear stability}

We consider the Brusselator model, long a paradigm for nonlinear analysis, given by

$$
\begin{gathered}
\frac{\partial f}{\partial \tau}=D_{f} \frac{\partial^{\alpha} f}{\partial|\xi|^{\alpha}}+E-(B+1) f+f^{2} g \\
\frac{\partial g}{\partial \tau}=D_{g} \frac{\partial^{\beta} g}{\partial|\xi|^{\beta}}+B f-f^{2} g, \quad \tau>0, \quad \xi \in \mathbb{R} .
\end{gathered}
$$

The diffusion coefficients $D_{f}, D_{g}$, the activator input rate $E$, and the control parameter $B$, are positive quantities. The action of the operator $\partial^{\gamma} / \partial|\xi|^{\gamma}$ in Fourier space is

$$
\mathscr{F}\left[\partial^{\gamma} u(\xi) / \partial|\xi|^{\gamma}\right](k)=-|k|^{\gamma} \mathscr{F}[u(\xi)](k), \quad 1<\gamma<2 .
$$

The equilibrium (basic) state of this system is $(f, g)=(E, B / E)$ for all values of the parameters.

Rescaling (2.1) and (2.2) using $f=E+u_{*} u, g=B / E+v_{*} v, \tau=t$, and $\xi=\ell_{*} x$, where $u_{*}=\left(D_{g} / D_{f}^{\beta / \alpha}\right)^{1 / 2}, v_{*}=1 / u_{*}$, and $\ell_{*}=D_{f}^{1 / \alpha}$, the Brusselator system becomes

$$
\begin{gathered}
\frac{\partial u}{\partial t}=\frac{\partial^{\alpha} u}{\partial|x|^{\alpha}}+(B-1) u+Q^{2} v+\frac{B}{Q} u^{2}+2 Q u v+u^{2} v \\
\eta^{2} \frac{\partial v}{\partial t}=\frac{\partial^{\beta} v}{\partial|x|^{\beta}}-B u-Q^{2} v-\frac{B}{Q} u^{2}-2 Q u v-u^{2} v,
\end{gathered}
$$

where $\eta=\sqrt{D_{f}^{\beta / \alpha} / D_{g}}>0, Q=E \eta>0$, and $x$ and $t$ represent the rescaled spatial and temporal variables, respectively. The equilibrium state is now at $u=v=0$.

To determine the stability of the critical point, we consider the normal mode solution, obtaining the dispersion relation between the growth rate $\sigma$ and the wave number $k>0, \eta^{2} \sigma^{2}+M_{1} \sigma+M_{2}=$ 0 , where $M_{1}=Q^{2}+k^{\beta}-\eta^{2}\left(B-1-k^{\alpha}\right)$, and $M_{2}=B Q^{2}+\left(k^{\beta}+Q^{2}\right)\left(1+k^{\alpha}-B\right)$.

Hopf bifurcation occurs if $M_{1}=0$ and $M_{2}>0$, which yields two pure imaginary eigenvalues. $M_{1}=0$ corresponds to $B=k^{\beta} / \eta^{2}+k^{\alpha}+1+Q^{2} / \eta^{2}$, which has a minimum, $B_{c r}^{(H)}=1+Q^{2} / \eta^{2}$ at $k=0$. The basic state is stable (unstable) for $B<B_{c r}^{(H)}\left(B>B_{c r}^{(H)}\right)$. In the unstable case, a spatially homogeneous oscillatory mode emerges. For $k=0$ and $B=B_{c r}^{(H)}$, the eigenvalue $\sigma=i Q / \eta \equiv i \omega$, where $\omega$ is the frequency of the oscillatory mode, while $\mathbf{c}^{\dagger}=\left(1, Q \eta^{2} /(Q+i \eta)\right)$ and $\mathbf{c}=\left(1,(i \eta-Q) /\left(Q \eta^{2}\right)\right)^{\top}$ are the left and right eigenvectors, respectively.

Turing instability occurs when $M_{2}=0$ and $M_{1}>0$, which yields $B=\left(Q^{2}+k^{\beta}\right)\left(1+k^{\alpha}\right) / k^{\beta}$. It has a single minimum $\left(k_{c r}, B_{c r}^{(T)}\right)$, given parametrically by

$$
B_{c r}^{(T)}=\frac{(1+z)^{2}}{1+(1-s) z}, \quad Q^{2}=\frac{s z^{1+1 / s}}{1+(1-s) z}, \quad k_{c r}=z^{1 / \alpha}
$$

where $s=\alpha / \beta$. Since $Q$ is real, we find that $0<z<\infty$ if $1 / 2<s \leq 1$, and $0<z<1 /(s-1)$ if $1<s<2$. The corresponding left and right eigenvectors of the zero eigenvalue are, respectively, 
$\mathbf{a}^{\dagger}=\left(1, s z \eta_{c}^{2} /(1+z)\right)$ and $\mathbf{a}=\left(z^{1 / s},-1-z\right)^{\top}$. For the Turing instability, a time-independent spatially periodic pattern may emerge with spatial wave number $k=k_{c r}$.

Turing and Hopf instability thresholds coincide at the C2THP where $B=B_{c r}^{(T)}=B_{c r}^{(H)} \equiv B_{c r}$, which occurs when $\eta=\eta_{c} \equiv \sqrt{s z^{1 / s} /(z+s+1)}$. Thus, as the control parameter $B$ is increased beyond $B_{c r}$, a Turing mode and a Hopf mode simultaneously bifurcate from the basic state, giving rise to terms of the form $A \mathbf{a} e^{i k_{c r} x}$ and $C \mathbf{c} e^{i \omega t}$ in $(u, v)^{\top}$. We note that $B_{c r}, Q, \eta_{c}$, and the activator input rate, $E=Q / \eta_{c}$, are increasing functions of $z$ for all allowed values of $z$.

\section{Weakly nonlinear analysis}

We analyze the system near the C2THP, i.e., let $\eta=\eta_{c}+\epsilon^{2} \eta_{2}(0<\epsilon \ll 1)$. If $\eta_{2}>0(<0)$, the Hopf (Turing) mode appears first as the parameter $B$ is increased. We interpret this as changing the parameter $E$, keeping $Q$ constant. Thus, changing $\eta_{2}$ will only affect the Hopf stability curve, not the Turing curve. Also, let $B=B_{c r}+\epsilon^{2} \mu$, where $\mu>0$ is a real $O(1)$ quantity. This leads to the presence of two time scales. The original time scale, $t$, appears with oscillation frequency $\omega$, while the slow time scale, $T=\epsilon^{2} t$, accounts for the slow-time evolution of the Turing and Hopf modes. The three relevant spatial scales are $x, X_{1}=\epsilon x$, and $X_{2 / \alpha}=\epsilon^{2 / \alpha} x$, where the scaling for $X_{2 / \alpha}$ is chosen under the condition that $\alpha<\beta$. If $\alpha>\beta$, the third spatial scale would instead be $X_{2 / \beta}$. While we consider both cases in Section 4, the explicit expressions are for $\alpha<\beta$.

With the relevant scales established, we allow for the possibility of both $A$ and $C$ to be functions of the slow time scale as well as the two long spatial scales. Then, since the Turing mode may be a function of all three spatial scales and the Hopf mode a function of the two long spatial scales, we require analogues of the chain rule to obtain expressions for how the operator $\partial^{\gamma} / \partial|x|^{\gamma}$ acts on $u$ and $v$. While nothing in the linear stability analysis of Section 2 prevents the Hopf mode from being a function of the two long spatial scales, solvability conditions discussed below in the weakly nonlinear analysis limit the Hopf mode dependence to $X_{2 / \alpha}$ only. Then, since the expression obtained by applying $d^{\gamma} / d|x|^{\gamma}$ to a function of the form $F\left(x, X_{1}, X_{2 / \alpha}\right)$ does not reduce to the expression obtained by applying the operator to a function of the form $G\left(X_{2 / \alpha}\right)$ simply by letting $\partial / \partial x=\partial / \partial X_{1}=0$, we decompose the solutions $u$ and $v$ into sums of functions of the form $F\left(x, X_{1}, X_{2 / \alpha}, t, T\right)$ and $G\left(X_{2 / \alpha}, t, T\right)$. Since $F$ accounts for all $x$-dependent terms, whether or not they depend on $X_{1}$ and/or $X_{2 / \alpha}$, while $G$ accounts for all $x$-independent terms, this decomposition captures all possible terms that can arise in $u$ and $v$. We utilize the product rule [12] for $1<\gamma<2$,

$$
\frac{d^{\gamma}(f g)}{d|x|^{\gamma}}=\sum_{j=0}^{\infty}\left(\begin{array}{l}
\gamma \\
j
\end{array}\right) \frac{d^{\gamma-j} f}{d|x|^{\gamma-j}} \frac{d^{j} g}{d x^{j}}
$$

to compute 


$$
\begin{aligned}
\frac{d^{\gamma} F\left(x, X_{1}, X_{2 / \alpha}\right)}{d|x|^{\gamma}}=\left(\frac{\partial^{\gamma}}{\partial|x|^{\gamma}}+\gamma \frac{\partial^{\gamma-1}}{\partial|x|^{\gamma-1}}\left(\epsilon \frac{\partial}{\partial X_{1}}+\epsilon^{2 / \alpha} \frac{\partial}{\partial X_{2 / \alpha}}\right)+\right. & \\
& \left.+\epsilon^{2} \frac{\gamma(\gamma-1)}{2} \frac{\partial^{\gamma-2}}{\partial|x|^{\gamma-2}} \frac{\partial^{2}}{\partial X_{1}^{2}}+\ldots\right) F\left(x, X_{1}, X_{2 / \alpha}\right)
\end{aligned}
$$

where we have discarded terms smaller than $O\left(\epsilon^{2}\right)$. The computation of $d^{\gamma} G\left(X_{2 / \alpha}\right) / d|x|^{\gamma}$ requires a simpler version of the chain rule, which gives $d^{\gamma} G / d|x|^{\gamma}=\epsilon^{2 \gamma / \alpha} d^{\gamma} G / d\left|X_{2 / \alpha}\right|^{\gamma}$, where $\gamma$ is either $\alpha$ or $\beta$.

Due to the fractional powers of $\epsilon$ in (3.1), we include fractional powers in the expansions of $u$ and $v$ :

$$
\left(\begin{array}{l}
u \\
v
\end{array}\right) \sim \epsilon\left(\begin{array}{l}
u_{1} \\
v_{1}
\end{array}\right)+\epsilon^{2 / \alpha}\left(\begin{array}{l}
u_{2 / \alpha} \\
v_{2 / \alpha}
\end{array}\right)+\epsilon^{2}\left(\begin{array}{l}
u_{2} \\
v_{2}
\end{array}\right)+\epsilon^{1+2 / \alpha}\left(\begin{array}{l}
u_{1+2 / \alpha} \\
v_{1+2 / \alpha}
\end{array}\right)+\epsilon^{3}\left(\begin{array}{l}
u_{3} \\
v_{3}
\end{array}\right)+\ldots
$$

We decompose $u_{i}$ and $v_{i}$ as

$$
\left(\begin{array}{c}
u_{i} \\
v_{i}
\end{array}\right)=\left(\begin{array}{c}
u_{i}^{(A)}\left(x, X_{1}, X_{2 / \alpha}, t, T\right) \\
v_{i}^{(A)}\left(x, X_{1}, X_{2 / \alpha}, t, T\right)
\end{array}\right)+\left(\begin{array}{c}
u_{i}^{(C)}\left(X_{2 / \alpha}, t, T\right) \\
v_{i}^{(C)}\left(X_{2 / \alpha}, t, T\right)
\end{array}\right)
$$

where we associate the letter $A$ with the Turing mode (though $u_{i}^{(A)}$ and $v_{i}^{(A)}$ also account for products of pure Turing and pure Hopf terms), and the letter $C$ with the Hopf mode. If $\alpha>4 / 3$, we must also include an $O\left(\epsilon^{4 / \alpha}\right)$ term in the expansion. Recalling the decomposition in (3.3), we substitute (3.2) into (2.4) and (2.5), and find that $u_{1}$ and $v_{1}$ satisfy

$$
\left(\frac{\partial}{\partial t}-\mathbf{D}_{0} \mathscr{D}-\mathbf{M}_{0}\right)\left(\begin{array}{c}
u_{1}^{(A)} \\
v_{1}^{(A)}
\end{array}\right)+\left(\frac{\partial}{\partial t}-\mathbf{M}_{0}\right)\left(\begin{array}{c}
u_{1}^{(C)} \\
v_{1}^{(C)}
\end{array}\right)=0
$$

where

$$
\mathbf{D}_{0}=\left(\begin{array}{cc}
1 & 0 \\
0 & \frac{1}{\eta_{c}^{2}}
\end{array}\right), \quad \mathscr{D} \equiv\left(\begin{array}{cc}
\frac{\partial^{\alpha}}{\partial|x|^{\alpha}} & 0 \\
0 & \frac{\partial^{\beta}}{\partial|x|^{\beta}}
\end{array}\right), \quad \mathbf{M}_{0}=\left(\begin{array}{cc}
B_{c r}-1 & Q^{2} \\
-\frac{B_{c r}}{\eta_{c}^{2}} & -\frac{Q^{2}}{\eta_{c}^{2}}
\end{array}\right)
$$

Thus

$$
\left(\begin{array}{l}
u_{1} \\
v_{1}
\end{array}\right)=A\left(X_{1}, X_{2 / \alpha} T\right) \mathbf{a} e^{i k_{c r} x}+C\left(X_{2 / \alpha}, T\right) \mathbf{c} e^{i \omega t}+\text { c.c. },
$$

where c.c. denotes complex conjugate. We have allowed only $A$ to depend on both long scales. If we had assumed that $C$ also depended on both long scales, $O\left(\epsilon^{\alpha}\right)$ and $O\left(\epsilon^{\beta}\right)$ terms would need to be included in (3.2). In this case, solvability conditions at $O\left(\epsilon^{1+\alpha}\right)$ and $O\left(\epsilon^{1+\beta}\right)$ would require that $C$ be independent of $X_{1}$. These are the solvability conditions mentioned above that dictate that 
the Hopf mode can only be a function of $X_{2 / \alpha}$. To see this, we apply the fractional operator to a function of the form $H\left(X_{1}, X_{2 / \alpha}\right)$ :

$$
\begin{aligned}
\frac{d^{\gamma} H\left(X_{1}, X_{2 / \alpha}\right)}{d|x|^{\gamma}}=\left(\epsilon^{\gamma} \frac{\partial^{\gamma}}{\partial\left|X_{1}\right|^{\gamma}}+\epsilon^{\gamma-1+2 / \alpha} \gamma \frac{\partial^{\gamma-1}}{\partial\left|X_{1}\right|^{\gamma-1}} \frac{\partial}{\partial X_{2 / \alpha}}+\right. & \\
& \left.+\epsilon^{\gamma-2+4 / \alpha} \frac{\gamma(\gamma-1)}{2} \frac{\partial^{\gamma-2}}{\partial\left|X_{1}\right|^{\gamma-2}} \frac{\partial^{2}}{\partial X_{2 / \alpha}^{2}}+\ldots\right) H\left(X_{1}, X_{2 / \alpha}\right)
\end{aligned}
$$

The presence of an $O\left(\epsilon^{\gamma}\right)$ term in (3.5) would require that we include terms of $O\left(\epsilon^{\alpha}\right), O\left(\epsilon^{\beta}\right)$, $O\left(\epsilon^{1+\alpha}\right)$, and $O\left(\epsilon^{1+\beta}\right)$ in the expansion of $u$ in (3.2), among terms of other orders. The right hand side of the $O\left(\epsilon^{1+\alpha}\right)$ equation would then contain a secular-producing term

$$
\left(\begin{array}{l}
1 \\
0
\end{array}\right) \frac{\partial^{\alpha} C}{\partial\left|X_{1}\right|^{\alpha}} e^{i \omega t}
$$

which is not orthogonal to $\mathbf{c}^{\dagger}$, and thus the solvability condition is not met. A similar violation of the solvability condition is also seen at $O\left(\epsilon^{1+\beta}\right)$, and also in the case of $\alpha=\beta$. To avoid this, we do not allow $C$ to depend on $X_{1}$.

The $O\left(\epsilon^{2 / \alpha}\right)$ equation is the same as the $O(\epsilon)$ equation, with $u_{2 / \alpha}$ and $v_{2 / \alpha}$ satisfying the same homogeneous equation as $u_{1}$ and $v_{1}$. Thus we may take $u_{2 / \alpha}=v_{2 / \alpha}=0$ without loss of generality (the same applies for $u_{4 / \alpha}$ and $v_{4 / \alpha}$ ).

While the left hand side of the $O\left(\epsilon^{2}\right)$ equation is the same as that in (3.4), its right hand side contains secular-producing terms proportional to $e^{i k_{c r} x}$. However, the solvability condition is satisfied (the secular-producing terms are orthogonal to $\mathbf{a}^{\dagger}$ ), which leads to the solution

$\left(\begin{array}{l}u_{2} \\ v_{2}\end{array}\right)=A^{2} \mathbf{p}_{2 s} e^{i 2 k_{c r} x}+C^{2} \mathbf{p}_{2 t} e^{i 2 \omega t}+A C \mathbf{p}_{L} e^{i \phi_{L}}+A C^{*} \mathbf{p}_{R} e^{i \phi_{R}}+|A|^{2} \mathbf{p}_{0 s}+|C|^{2} \mathbf{p}_{0 t}+\mathbf{p}_{s} e^{i k_{c r} x}+c . c .$, where $\phi_{L}=k_{c r} x+\omega t$ and $\phi_{R}=k_{c r} x-\omega t$.

The $O\left(\epsilon^{1+2 / \alpha}\right)$ equation, like the $O\left(\epsilon^{2}\right)$ equation, contains secular-producing terms orthogonal to $\mathbf{a}^{\dagger}$. However, while $u_{1+2 / \alpha}$ and $v_{1+2 / \alpha}$ are non-zero, they do not enter the $O\left(\epsilon^{3}\right)$ equation. Upon solving for the vectors $\mathbf{p}_{2 s}, \mathbf{p}_{2 t}$, etc., and applying the solvability condition at $O\left(\epsilon^{3}\right)$, we obtain, upon rescaling, the amplitude equations

$$
\begin{gathered}
\frac{\partial A}{\partial T}=A+\frac{\partial^{2} A}{\partial X_{1}^{2}}+\zeta A|A|^{2}+\psi_{2} A|C|^{2} \\
\frac{\partial C}{\partial T}=\rho C+\left(\alpha_{1}+i \alpha_{2}\right) \frac{\partial^{\alpha} C}{\partial\left|X_{2 / \alpha}\right|^{\alpha}}+\left(-\left|\beta_{1}\right|+i \beta_{2}\right) C|C|^{2}+\left(\delta_{1}+i \delta_{2}\right) C|A|^{2},
\end{gathered}
$$

where $\zeta= \pm 1$, depending on the values of $\alpha, \beta$, and $z$, while $\psi_{2}, \alpha_{1}, \alpha_{2}, \beta_{1}, \beta_{2}, \delta_{1}$, and $\delta_{2}$, are real functions of $\alpha, \beta$, and $z$. The coefficient $\rho$, while also real, is a function of $\alpha, \beta$, and $z$, as well as $\mu$ and $\eta_{2}$. Finally, it can be shown that $\alpha_{1}>0$. 
We restrict $z$ to the interval $I$ such that $\zeta=-1$, i.e., there exists nonlinear saturation of the Turing mode. We also note that (3.6) and (3.7) were derived under the necessary condition that $C$ be independent of $X_{1}$. Since (3.7) contains terms involving both $A$ and $C,|A|$ must be spatially homogeneous. Thus, (3.6) and (3.7) describe only amplitudes $A$ whose dependence on $X_{1}$ takes the form $e^{i h\left(X_{1}\right)}$ for a real function $h$. Since $A$ can depend on both $X_{1}$ and $X_{2 / \alpha}$, there are no restrictions on the way in which $C$ can depend on $X_{2 / \alpha}$.

Lastly, the techniques used here resemble those used for the regular diffusion Brusselator model. There are, however, important differences, one being that two long spatial scales are present instead of the single long scale $X_{1}$ in the regular model. Secondly, the expansions of $u$ and $v$ include fractional orders of $\epsilon$, whereas only integer powers were required in the regular model. Thirdly, the rules of differentiation require that the solution be decomposed into separate functions that depend differently on the relevant variables. The resulting form of the amplitude equations are also different in that the equation for $C$ is now an integro-differential equation. A shorter version of this section is presented in [14].

\section{Solutions of the amplitude equations and their long-wave in- stabilities}

In this section, we seek special solutions of (3.6) and (3.7), namely a pure Turing solution, a pure Hopf solution, and a mixed mode solution. We then study the instabilities of these solutions to long-wave perturbations. We first consider the pure Turing solution, given by $C=0$ and $A=\tilde{A} e^{i K_{A} X_{1}}$ with $\tilde{A}=\left(1-K_{A}^{2}\right)^{1 / 2}$. To study its stability, we linearize around it using $A=$ $\left(\tilde{A}+a\left(X_{1}, T\right)\right) e^{i K_{A} X_{1}}$, and $C=c\left(X_{2 / \alpha}, T\right)$. The resulting linearized equations decouple, so we analyze each separately. A long-wave perturbation $a\left(X_{1}, T\right)$ yields the familiar Eckhaus stability criterion

$$
\left|K_{A}\right|<\frac{1}{\sqrt{3}}
$$

If (4.1) is not satisfied, the perturbation grows, changing the spatial frequency of the solution. A long-wave perturbation $c\left(X_{2 / \alpha}, T\right)$ with wave number $k \ll 1$ results in the dispersion relation $\sigma=\rho-\alpha_{1}|k|^{\alpha}+\tilde{A}^{2} \delta_{1} \pm\left. i\left|-\alpha_{2}\right| k\right|^{\alpha}+\tilde{A}^{2} \delta_{2} \mid$, whose real part must be negative for long-wave stability. If the real part is positive, the perturbation grows, changing the spatial structure of the solution and also introducing a time-oscillatory component. The long-wave stability criterion is $\rho+\tilde{A}^{2} \delta_{1}<0$. If $\delta_{1}<0$, long-wave perturbations of the Hopf amplitude decay for all $\rho<0$, or even if $\rho>0$ as long as $\rho$ remains sufficiently small. If $\delta_{1}>0$, long-wave perturbations of the Hopf amplitude can grow even for $\rho<0$, as long as $\rho$ is sufficiently close to 0 .

For the regular diffusion model, $\delta_{1}>0$ for $z \lesssim 0.26$, or equivalently, $\eta_{c} \lesssim 0.34$, meaning that the inhibitor $(g)$ diffuses significantly faster than the activator $(f)$. In the anomalous model, we obtain an analogous result for $\alpha$ and $\beta$, since these two parameters have a greater impact on the rate of diffusion than do the diffusion coefficients. In contrast to the regular model, $\delta_{1}$ can 
be positive even if $\alpha<\beta$, that is, if the inhibitor diffuses more slowly than the activator. For $\alpha \lesssim 1.65, \delta_{1}<0$ for all $z \in I$, meaning that sufficiently fast diffusion of the activator makes it impossible for long-wave perturbations of the Hopf mode to grow if $\rho<0$. This behavior is not seen in the regular model.

Next, we consider the pure Hopf solution, given by $A=0$ and $C=\tilde{C} e^{i K_{C} X_{2 / \alpha}+i \Omega T}$ with $\tilde{C}=\left(\left(\rho-\alpha_{1}\left|K_{C}\right|^{\alpha}\right) /\left|\beta_{1}\right|\right)^{1 / 2}$ and $\Omega=-\alpha_{2}\left|K_{C}\right|^{\alpha}+\beta_{2} \tilde{C}^{2}$. We note that, since the quantity $\rho-\alpha_{1}\left|K_{C}\right|^{\alpha}$ must be positive, $\rho$ must be positive for the pure Hopf solution to exist. Long-wave perturbations of the form $e^{\sigma T+i k X_{2 / \alpha}}$ yield two growth rates, one of which is negative, the other of which has the expansion for small $k, \sigma=a_{1} k+a_{2} k^{2}+O\left(k^{3}\right)$, where

$$
a_{1}=-i \frac{\alpha\left(\alpha_{1} \beta_{2}+\alpha_{2}\left|\beta_{1}\right|\right)\left|K_{C}\right|^{\alpha-1}}{\left|\beta_{1}\right|}
$$

and

$$
a_{2}=\frac{\alpha(\alpha-1)\left(\alpha_{2} \beta_{2}-\alpha_{1}\left|\beta_{1}\right|\right)}{2\left|\beta_{1}\right|}+\frac{\alpha^{2} \alpha_{1}^{2}\left(\beta_{1}^{2}+\beta_{2}^{2}\right)\left|K_{C}\right|^{\alpha}}{2\left|\beta_{1}\right|^{3} \tilde{C}^{2}} .
$$

Requiring $a_{2}<0$ for stability leads to the generalized Eckhaus criterion,

$$
\left|K_{C}\right|^{\alpha}<\frac{\rho}{R \alpha_{1}}
$$

where

$$
R=1+\frac{\alpha \alpha_{1}\left(\beta_{1}^{2}+\beta_{2}^{2}\right)}{(\alpha-1)\left|\beta_{1}\right|\left(\alpha_{1}\left|\beta_{1}\right|-\alpha_{2} \beta_{2}\right)} .
$$

Thus, if (4.2) is not satisfied, both the spatial and temporal structures of the solution are altered as a long-wave perturbation grows with amplitude oscillating at a frequency different from $\Omega$. As in the regular diffusion case, the magnitude of $R$ is greater than unity for all $z \in I$. However, if $\alpha<\beta$, unlike the regular diffusion case, $R$ is positive only for $z$ sufficiently small. Beyond this interval, $R$ becomes negative so that (4.2) is never satisfied, in which case the pure Hopf solution must be long-wave unstable. Restricting $z$ to sufficiently small values for which $R$ is positive implies that $B_{c r}, Q, \eta_{c}$, and $E$, must all be sufficiently small. For example, $\eta_{c}$ must be less than $\sim 0.62$, and thus, while the rate of diffusion is dominated by the diffusion exponents $\alpha$ and $\beta, \eta_{c}$ still impacts whether or not the Hopf mode can be long-wave stable. Note, however, that $\eta_{c}$ is not a strict comparison between the diffusion coefficients $D_{f}$ and $D_{g}$, as these parameters do not even have the same units. The qualitative behavior of $R$ in the $\alpha>\beta$ case is the same as for regular diffusion, where $R>1$ for all $z \in I$, suggesting that faster diffusion of the activator versus the inhibitor may contribute to instability of the pure Hopf solution.

Finally we consider the mixed mode solution, given by $A=\tilde{A} e^{i K_{A} X_{1}}$ and $C=\tilde{C} e^{i K_{C} X_{2 / \alpha}+i \Omega T}$ with

$$
\tilde{A}=\left(\frac{\psi_{2}\left(\rho-\alpha_{1}\left|K_{C}\right|^{\alpha}\right)+\left|\beta_{1}\right|\left(1-K_{A}^{2}\right)}{\Delta}\right)^{1 / 2}, \quad \tilde{C}=\left(\frac{\rho-\alpha_{1}\left|K_{C}\right|^{\alpha}+\delta_{1}\left(1-K_{A}^{2}\right)}{\Delta}\right)^{1 / 2}
$$


where $\Delta=\left|\beta_{1}\right|-\psi_{2} \delta_{1}$, and $\Omega=-\alpha_{2}\left|K_{C}\right|^{\alpha}+\beta_{2} \tilde{C}^{2}+\delta_{2} \tilde{A}^{2}$. Of course, we must restrict $K_{A}$ and $K_{C}$ so that $\tilde{A}$ and $\tilde{C}$ are real. Linearizing (3.6) and (3.7) around this mixed mode solution with small perturbations $a\left(X_{2 / \alpha}, T\right)$ and $c\left(X_{2 / \alpha}, T\right)$ results in the coupled equations

$$
\frac{\partial a}{\partial T}=\left(1-K_{A}^{2}\right) a-\tilde{A}^{2}\left(a^{*}+2 a\right)+\psi_{2}\left[\tilde{A} \tilde{C}\left(c^{*}+c\right)+a \tilde{C}^{2}\right]
$$

and

$$
\begin{aligned}
\frac{\partial c}{\partial T}= & -i \Omega c+\rho c+\left(\alpha_{1}+i \alpha_{2}\right)\left(-\left|K_{C}\right|^{\alpha} c+i \alpha\left|K_{C}\right|^{\alpha-1} \frac{\partial c}{\partial X_{2 / \alpha}}+\frac{\alpha(\alpha-1)}{2}\left|K_{C}\right|^{\alpha-2} \frac{\partial^{2} c}{\partial X_{2 / \alpha}^{2}}\right) \\
& +\left(-\left|\beta_{1}\right|+i \beta_{2}\right) \tilde{C}^{2}\left(c^{*}+2 c\right)+\left(\delta_{1}+i \delta_{2}\right)\left[\tilde{A} \tilde{C}\left(a^{*}+a\right)+c \tilde{A}^{2}\right]
\end{aligned}
$$

Eqs. (4.3) and (4.4) contain terms involving both $a$ and $c$, and so, for consistency, we require $a$ to depend only on $X_{2 / \alpha}$. We consider two types of perturbations: spatially homogeneous perturbations of a spatially dependent solution $\left(K_{A}, K_{C} \neq 0\right)$, and long-wave perturbations of a spatially homogeneous solution $\left(K_{A}=K_{C}=0\right)$. For the first case, the resulting dispersion relation yields two zero eigenvalues and 2 negative eigenvalues as long as $\Delta>0$, with one of the eigenvalues turning positive if $\Delta<0$. If $\Delta<0$, the solution decays to either a pure Turing or pure Hopf mode, depending on the initial conditions [6], changing the temporal and spatial structures of the solution. Thus, a necessary (and sufficient) condition for stability of the spatially dependent mixed mode solution to homogeneous perturbations is $\Delta>0$. As in the regular case, there are values of $\alpha$ and $\beta$ for which stability is possible for both sufficiently large and small values of $z$. These occur for $(\alpha, \beta)$ pairs that are near $(2,2)$. Sufficiently small (large) $z$ refers to an interval of $z$ that ranges from the smallest (largest) $z \in I$ to some larger (smaller) $z \in I$. For $(\alpha, \beta)$ pairs where $\alpha$ is sufficiently large, it is possible that $\Delta>0$ only for $z$ sufficiently small. More specifically, for all such $(\alpha, \beta)$ pairs, stability is possible only if $\eta_{c} \lesssim 0.42$. Similarly, for all $(\alpha, \beta)$ for which stability is only possible for sufficiently large $z$, stability is possible only if $\eta_{c} \gtrsim 0.65$. For some $(\alpha, \beta)$ pairs with $\beta$ sufficiently small, stability is impossible.

For the spatially homogeneous solution, taking $K_{A}=K_{C}=0$, (4.3) remains the same, while the derivative term in (4.4) is replaced by $\partial^{\alpha} c / \partial\left|X_{2 / \alpha}\right|^{\alpha}$. Upon inserting long-wave perturbations of the form $e^{\sigma T+i k X_{2 / \alpha}}$, the resulting dispersion relation yields two zero eigenvalues and one negative eigenvalue as long as $\Delta>0$, while the fourth eigenvalue has the expansion for small $k$, $\sigma \sim a_{\alpha}|k|^{\alpha}$, where

$$
a_{\alpha}=\frac{\alpha_{2}\left(\beta_{2}+\psi_{2} \delta_{2}\right)-\alpha_{1} \Delta}{\Delta} .
$$

Thus, long-wave stability of the spatially homogeneous solution requires $\Delta>0$ and $a_{\alpha}<0$. If either one of these conditions is not satisfied, a long-wave spatial pattern appears, breaking the spatial homogeneity. Like the regular diffusion case, there exist $(\alpha, \beta)$ pairs such that stability is possible only for sufficiently large $z$. More specifically, for all such $(\alpha, \beta)$ pairs, long-wave stability of the spatially homogeneous mixed mode solution is possible only if $\eta_{c} \gtrsim 0.65$. These occur 
for $\alpha$ sufficiently close to $\beta$, but only for $\alpha>\beta$. As in the pure Hopf stability analysis, it appears that the $\alpha>\beta$ case more closely resembles regular diffusion in terms of stability properties. For $\alpha<\beta$ with $\alpha$ and $\beta$ sufficiently large, stability is possible only for $z$ sufficiently small. For all such $(\alpha, \beta)$ pairs, $\eta_{c} \lesssim 0.37$. For both mixed mode solutions, $\alpha$ and $\beta$ determine whether or not there exist values of parameters, such as $\eta_{c}$, for which stability is possible, as for many $(\alpha, \beta)$ pairs, stability is impossible.

In summary, the evolution equations (3.6) and (3.7) appear similar to their regular diffusion counterparts, but differ both in the behavior of their coefficients, as well as their overall form, as (3.7) reflects non-local effects. As a result, the stability criteria differ greatly from those of regular diffusion. In the stability analysis of the pure Turing solution, there exist $(\alpha, \beta)$ such that longwave perturbations of the Hopf mode cannot grow if $\rho<0$ for any value of $z$. This is contrary to the regular diffusion case, for which growth is possible if the inhibitor diffuses sufficiently faster than the activator. Further, we found that there exist $(\alpha, \beta)$ for which long-wave perturbations of the Hopf mode can grow for $\rho<0$ even if the inhibitor diffuses more slowly than the activator. We also found that, for $\alpha<\beta$, there exist values of $z \in I$ such that stability of the pure Hopf solution is impossible, while for $\alpha>\beta$, stability criteria remains qualitatively similar. Finally, for the mixed mode, there exist $(\alpha, \beta)$ pairs sufficiently close to $(2,2)$ for which stability requirements are similar to those of regular diffusion. Away from this regime, these requirements can either change or stability may simply not be possible.

\section{Numerical results}

The system (2.4) and (2.5) was solved on the interval $0<x<L$ with periodic boundary conditions using Fourier spectral methods in space and a second order Runge-Kutta method in time. Differentiation in spectral space was computed using (2.3). We computed solutions in two regimes: near the stability threshold to confirm the analysis in Section 4, and far in the nonlinear regime to find solutions not predicted by weakly nonlinear analysis. When confirming the stability analysis of Section 4, a system length $L$ was employed so that the chosen initial conditions would be periodic. To determine long-wave stability of the solutions described in Section 4, we set as the initial conditions the respective solution plus a small long-wave perturbation. The Fourier spectrum of the initial condition thus contained a non-zero amplitude associated with the first order solution, and comparatively smaller amplitudes associated with the long-wave perturbations. The parameter $\mu$ was set to be an $O(1)$ quantity, as was $\eta_{2}$. In the cases for which the solution was long-wave stable, the Fourier amplitude of the first order solution remained constant to within $\sim O\left(\epsilon^{2}\right)$ of the initial (predicted) amplitude, while the amplitudes of the long-wave perturbations decayed. In cases for which the solution was long-wave unstable, the amplitude of the first order solution remained near its initial value for a time of $O\left(1 / \epsilon^{2}\right)$ before beginning to decay. The amplitude of the long-wave perturbation saw a slow initial decay that lasted for a time of $O\left(1 / \epsilon^{2}\right)$ before growing to the same order of magnitude as the amplitude of the first order solution. In this way, we were able to confirm results (4.1) and (4.2). Specifically, if the wavenumber of the pure Turing mode lies within the 
Eckhaus stable region, a pure Turing solution with that wavenumber is found numerically, while if the spatial wavenumber of the Hopf bifurcated solution lies within the generalized Eckhaus stable region, the Hopf bifurcated solution with that wavenumber is found numerically, thus confirming the results of the weakly nonlinear analysis. As a further corroboration of the weakly nonlinear analysis, in Fig. 1 we compare the oscillation frequency of the Hopf solution computed numerically to that predicted by the weakly nonlinear analysis, as a function of the bifurcation parameter $\mu$. We see that for small $\mu$ (weakly nonlinear case) there is excellent agreement between the two.

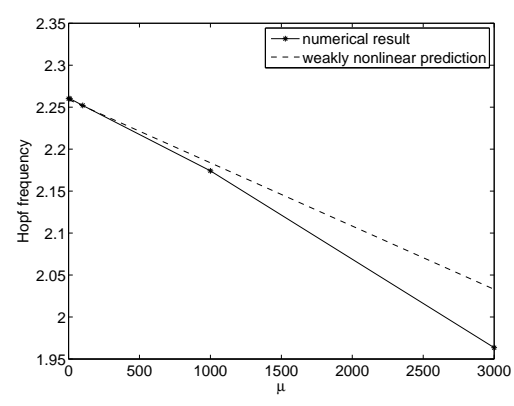

Figure 1: Comparison of weakly nonlinear prediction and numerical results of the frequency of spatially homogeneous oscillations as a function of the bifurcation parameter $\mu$. The parameters are $\alpha=1.4, \beta=1.5, z=1.6$, and $\eta_{2}=1$.

Results that involved components of both the Turing and Hopf modes were too computationally intensive to check. In particular, results pertaining to long-wave stability of the mixed mode were numerically inconclusive, as it appeared that a pure stable mixed mode exists only for $\epsilon$ too small to feasibly compute a steady state solution. However, it was observed that if $\Delta<0$, a solution that started out with both a Turing and Hopf mode decayed to either a pure Turing or pure Hopf solution, depending on the values of the parameters. If $\Delta>0$, both modes remained present for the entire length of the computation, though the respective amplitudes were not constant, indicating that the steady state was not that of a pure mixed mode.

In the sections below, we discuss results of computations with $\mu$ of $O\left(1 / \epsilon^{2}\right)$ so that the system is in the fully nonlinear regime. To reach what was determined to be a steady state, the system was evolved over $1.5 \times 10^{4}$ units of time. To verify that the time period was sufficiently long, for some results, we compared solutions obtained near $t=1.5 \times 10^{4}$ with those obtained near $t=3 \times 10^{4}$ to ensure that the solutions obtained were in fact in steady state. In such cases, no qualitative differences were observed between the solutions at the two times. The Fourier spectrum of the steady state solutions were also monitored to ensure that the amplitudes in the tail of the spectrum did not exceed $O\left(10^{-3}\right)$ of that of magnitudes of the most dominant modes, thus indicating that aliasing was not significant for the computational results presented here. In all space-time plots presented, the spatial variable $x$ runs horizontally while time runs vertically. 


\subsection{The fully nonlinear regime with equal diffusion exponents}

Taking $\mu$ to be of $O\left(1 / \epsilon^{2}\right)$, we computed solutions not predicted by the weakly nonlinear analysis, in the fully nonlinear regime of the regular and superdiffusive Brusselator models. The parameter $\eta_{2}$ was still kept as an $O(1)$ quantity so that the system remained near the C2THP. We consider only $\alpha=\beta$ in this subsection. The following subsection below discusses the $\alpha \neq \beta$ case. Figures 2 - 11 show space-time plots of $u$, or plots of $u(x)$ at particular instants of time, in steady states with $\alpha=\beta$, starting from random initial conditions and setting the parameters $\epsilon^{2} \mu=1, \eta_{2}=1$, and $L=500$ while varying $z$. Since it appears that different initial conditions evolve to different steady states (cf. [5] for regular diffusion), we computed steady states from different random initial conditions for each set of parameters. Thus, for each $(\alpha, \beta)=(1.1,1.1),(1.5,1.5)$ and $(2,2)$, we computed steady states with $z$ ranging from 0.2 to 3 in increments of 0.2 . The parameters $L, \mu$ and $\eta_{2}$ were kept constant. For each $(\alpha, \beta, z)$ parameter set, we computed steady states from the same set of random initial conditions (i.e., the random initial conditions were generated in such a way that they were reproducible, and thus could be used again). Note that if $L$ is lowered significantly, many of the steady state patterns disappear.

For $z$ small $(\sim 0.2)$, setting $(\alpha, \beta)=(1.1,1.1),(1.5,1.5)$ and $(2,2)$, we found that all initial conditions employed resulted in a steady state consisting only of stationary stripes (a pure Turing steady state), with the dominant wave number near $L z^{1 / \alpha} /(2 \pi)$, depending on the initial conditions. For $z=0.4$, we find that the $(1.1,1.1)$ case still yielded a pure Turing solution for all initial conditions tested. The $(1.5,1.5)$ case yielded some pure Hopf steady states (spatially homogeneous oscillations) and some pure Turing states, depending on the initial conditions, while the $(2,2)$ case yielded a pure Hopf solution for all initial conditions. For $z=0.6$, both the $(1.5,1.5)$ and $(2,2)$ cases yielded pure Hopf steady states of the same frequency, while the $(1.1,1.1)$ case yielded both pure Turing and pure Hopf steady states, depending on the initial conditions. Thus, anomalous diffusion with equal diffusion exponents delays the development of Hopf behavior (in terms of increasing $z$ ). For $z>2$, all three cases yielded pure Turing steady states for all random initial conditions tested. For $1.2 \leq z \leq 1.8$, we found spatiotemporal patterns for either one or both of the $(1.5,1.5)$ and $(2,2)$ cases. For the values of $z$ and the random initial conditions tested, we did not find any spatiotemporal patterns for the $(1.1,1.1)$ case.

In the descriptions below, we will use the term "spot" to denote a local maximum of $u$. We will say that a spot is created when such a maximum forms, and that a spot is annihilated when the maximum disappears. We will also say that two spots that propagate away from each other are counter propagating, and that two spots that propagate toward each other are oppositely propagating. In a system with periodic boundary conditions, these definitions might appear to be confusing, as spots which are created as counter propagating can theoretically, after rotating through a full period, become oppositely propagating. However, this does not occur in our computations because the spots are prevented from going all the way around the full period by the presence of obstacles, e.g., regions of stationary stripes which halt the motion of the spots by absorbing them. These spots account for the time-oscillatory regions in the spatiotemporal patterns described below, which, in addition, often also contain stripe-like regions.

Figures 2(a) - 2(c) show spatiotemporal patterns with regular diffusion, $B=5.84$ and $\eta=$ 


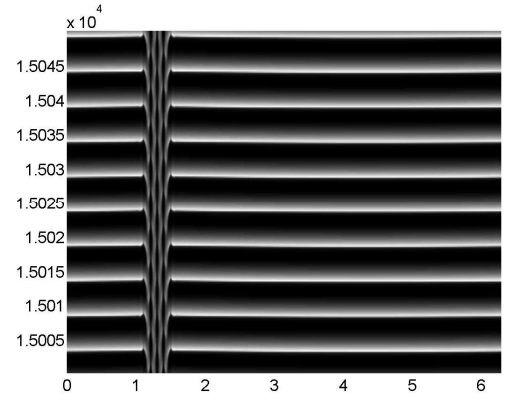

(a)

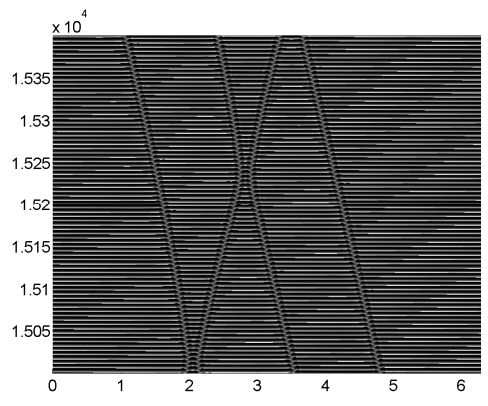

(c)

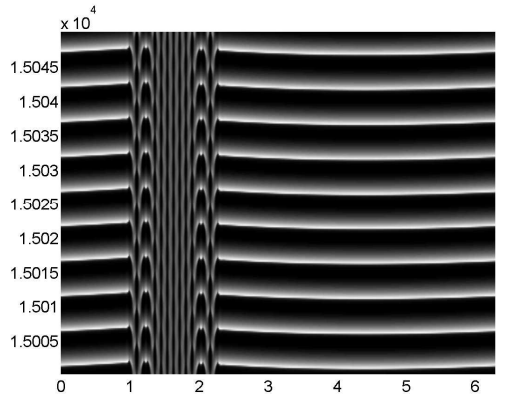

(b)

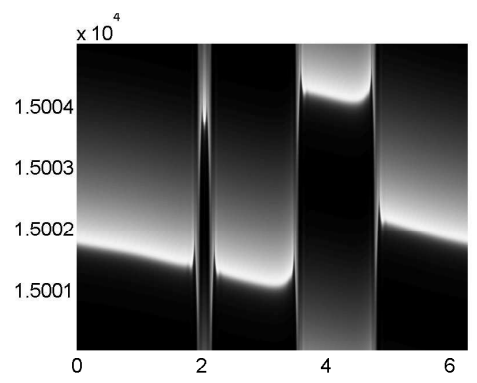

(d)

Figure 2: Spatiotemporal patterns of $u$ for regular diffusion with $z=1.2, B=5.84$ and $\eta=0.612$. The figures differ only in the random initial condition employed. Most of the patterns for this parameter set have low spatial frequency and are mainly time-oscillatory, with smaller intervals of stationary or breathing stripes. Figure (c) shows multiple propagating dislocations. Light colors indicate more positive values of $u$, while dark colors indicate less positive values of $u$. Figure (d) shows a small interval in time of (c), corresponding to the time interval depicted in Figures 6(a) to 6(i). The space-time plots of $v$ are essentially the same, except with the colors inverted.

0.612 for different random initial conditions. Figure 2(a) shows breathing stripes embedded in a time-oscillatory and nearly spatially homogeneous structure. The stripes breathe while the values of the minima and maxima also oscillate in time. The dynamical behavior of this mode is illustrated in more detail in Figures 3(a) though 3(e) where we plot $u$ versus $x$ for selected times near the first (lowest) horizontal stripe in Figure 2(a). The apparently horizontal stripes are in fact slightly Ushaped, as a spot is created near $x=4.5$ (spot A in Figures 3(a) and 3(b)), which splits into two spots that counter propagate toward opposite sides of the stripes (spots B and C in Figure 3(c)), after which $u$ decays until the start of the next event. The rapid rise and gradual decay of $u$ indicates temporal relaxation oscillations (illustrated by a plot of $u$ at $x=4.5$ in Figure 4). In the early stages of the decay, the outer spot on either side of the striped region is absorbed into the 
incoming spot (Figure 3(d)), while in the latter stages of the decay (Figure 3(e)), two new spots are formed at the edge of the stripe region in place of those previously absorbed.

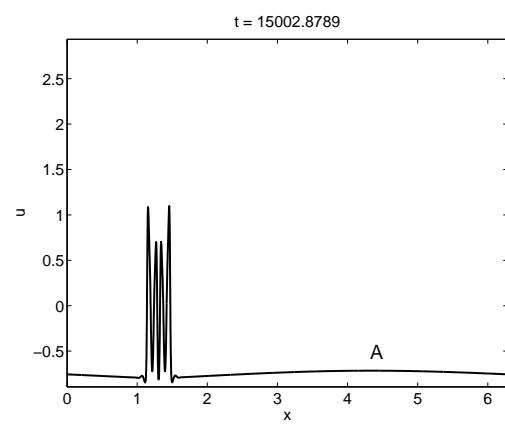

(a)

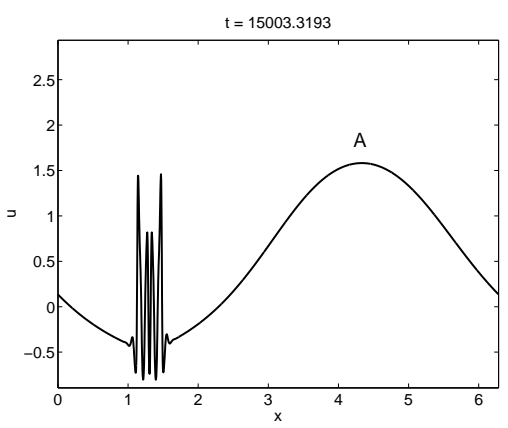

(b)

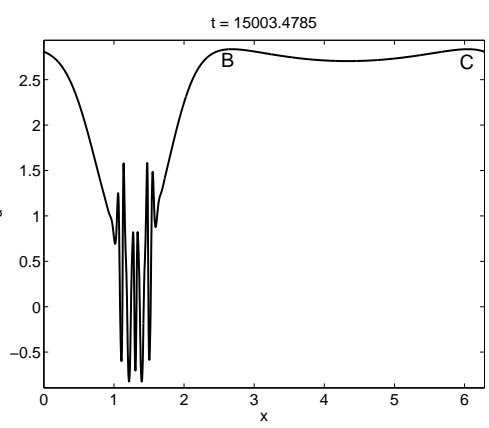

(c)

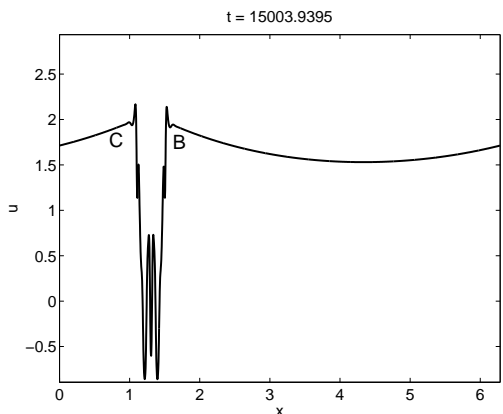

(d)

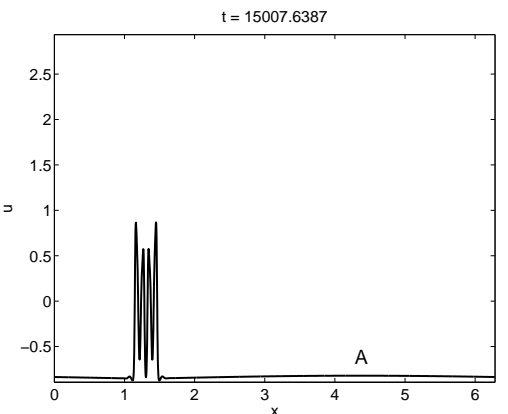

(e)

Figure 3: Plots of $u(x)$ at specific instants of time illustrating the creation and annihilation of spots in Figure 2(a). A spot (A) begins to form at $x \simeq 4.5$ in (a), which then grows ((b)) until it splits into two spots (B and $\mathrm{C}$ in (c)). The two spots are annihilated with the two spots straddling the striped region and decay $((\mathrm{d}))$ before the process repeats $((\mathrm{e}))$.

Figure 2(b) has qualitative similarities to Figure 2(a) in that one spot splits into two counter propagating spots. The U-type behavior is more pronounced and clearly visible in Figure 2(b) than in Figure 2(a). The way that the two spots interact with the boundary of the stripes is, however, the same as in Figure 2(a). A slight difference is that the spot creation site is slightly closer to the right side of the stripes. As a result, the spot traveling toward the right side of the stripes is annihilated before the one traveling to the left side. However, the main difference is that Figure 2(b) also has inverted U-shaped patterns embedded in the stripes, each of which corresponds to two spots created at the stripes that grow slowly and propagate toward one another. The dynamical behavior of this mode is illustrated in Figures 5(a) - 5(e) where we plot $u$ as a function of $x$ for times near the lower part of Figure 2(b). These figures are plotted only over the right end of the striped region, 


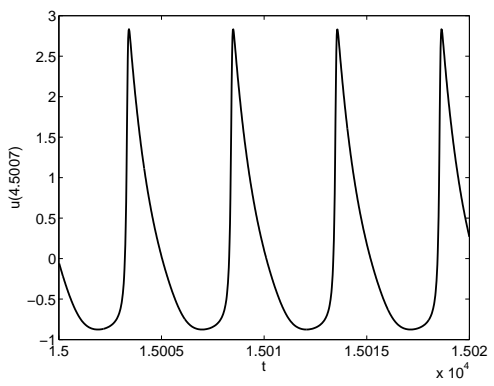

Figure 4: Plot of $u$ at $x=4.5$ as a function of time, corresponding to the bottom four stripes of Figure 2(a).

focusing on the inverted $\mathrm{U}$ structure $(x \simeq 1.9$ to $x \simeq 2.15)$. Two spots are centered at $x \simeq 1.95$ and $x \simeq 2.1$ and are labeled $\mathrm{B}$ and $\mathrm{C}$, respectively. A spot in the middle (spot A in Figure 5(a)) grows at a rate faster than the two oppositely propagating spots (Figure 5(b)) and achieves a greater maximal value than the two oppositely propagating spots (Figure 5(c)), accounting for the bright spots at the peaks of the inverted U's in Figure 2(b). As the three spots decay together, the two outside spots are eventually absorbed into the larger interior spot (Figure 5(d)) as two new spots form in their place (spots $\mathrm{B}^{\prime}$ and $\mathrm{C}^{\prime}$ in Figure 5(d) and Figure 5(e)). Just as the two spots that are annihilated at the stripes are out of phase, so too are the oscillations of the two inverted U's.

Figure 2(c) shows four dislocations that propagate uniformly in time, with three that propagate to the left, and one that propagates to the right. These dislocations are sites at which a spot is born on one side and propagates away from it, and another spot generated at an adjacent dislocation is annihilated on the other side. The four dislocations divide the system into four regions and act as boundaries where spots are born and annihilated. The dislocations appear to propagate until meeting another dislocation, at which point the two appear to repel each other. The speed of the dislocations (the inverse of the absolute value of the slopes of the dislocations shown in Figure 2(c)) appears to be constant in time and the same for all dislocations. The time at which a spot is created in one particular region is much closer to the time at which a spot is created in the interval on the other side of its neighbor. For example, at $t=1.5 \times 10^{4}$ in Figure 2(c), a spot creation event in the interval centered at $x \simeq 2$ will either be closely preceded or followed by a spot creation event in the interval centered at $x \simeq 4$.1. Figures 6(a) - 6(f) indicate the intervals (labeled 1-4 in the figures) and illustrate the dynamical behavior within each interval. The figures represent the dynamical evolution of the pattern for times near the lower part of Figure 2(c). Figure 6(a) shows the two regions centered at $x \simeq 2$ (region 2) and $x \simeq 4.1$ (region 4 ) near the end of a decay process, just as a spot has formed in the region centered at $x \simeq 3$ (region 3). This spot then grows (Figure 6(b)) and propagates to the left (Figure 6(c)) just as a spot begins to form to the left of $x=2$ in the region centered near $x \simeq 0$ (region 1 ). This spot grows and propagates to the left as the spot in region 3 is annihilated and the whole interval begins to decay (Figure 6(d)). The spot in region 1 continues to propagate to the left until it is annihilated near $x=5$ (Figure 6(e)). As intervals 1 and 3 decay, spots begin to form in regions 2 and 4 (Figure 6(f)). The spot in region 2 reaches its 


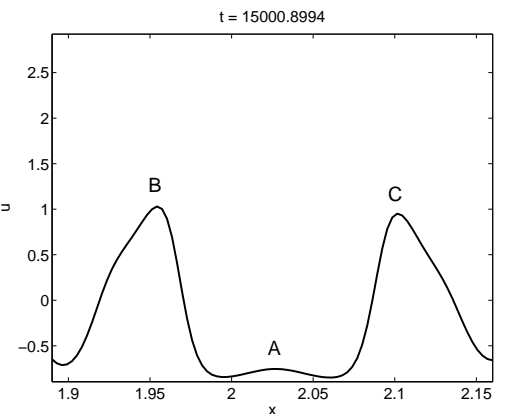

(a)

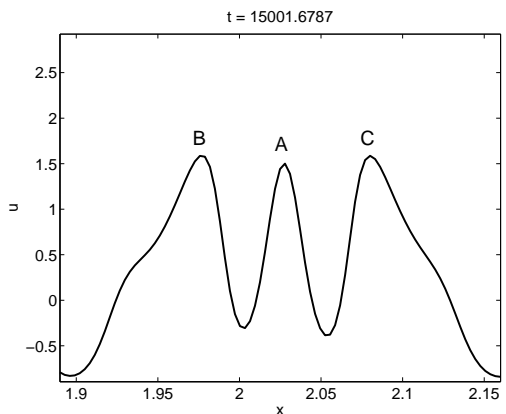

(b)

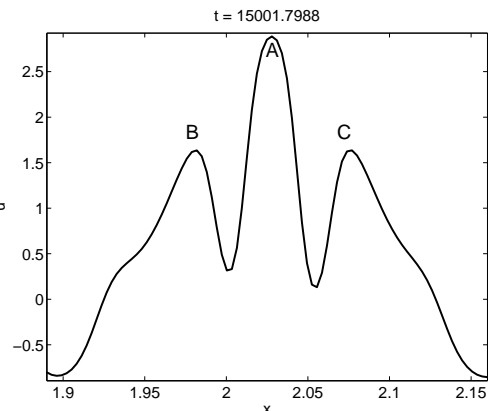

(c)

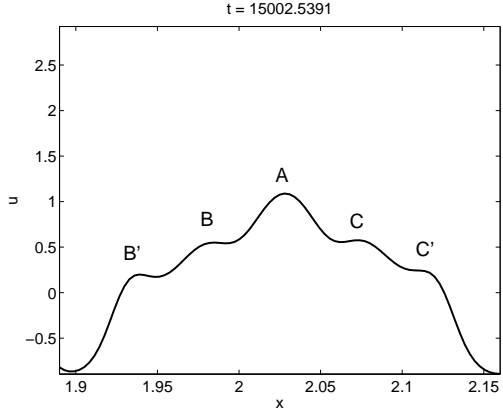

(d)

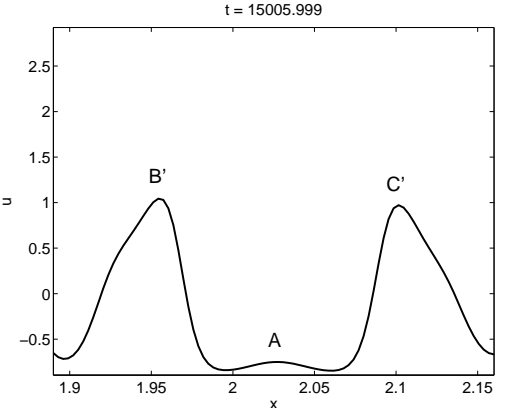

(e)

Figure 5: Line plots describing details of the right inverted U of Figure 2(b) at specific instants of time. A spot centered at $x \simeq 2.03$ grows at a faster rate than the two oppositely propagating spots centered at $x \simeq 1.95$ and $x \simeq 2.1$ ((a) and (b)). In (c), all three spots have achieved values close to their maximal value, with that of the middle spot being much larger. The three spots then decay together, appearing to merge together into one structure $((\mathrm{d}))$, from which two new spots are born to replace the two outer spots ((e)).

maximum (Figure 6(g)) before the spot in region 4 forms and propagates to the left (Figure 6(h) and 6(i)). Regions 2 and 4 then decay, and the process starts over again.

With $\alpha=\beta=1.5$ and $z=1.2$, the only steady states found using the same initial conditions as those used for the regular diffusion computations were those of spatially homogeneous oscillations.

For $z=1.4, B=6.76$, and $\eta=0.642$ (Figures 7(a) - 7(f)), we found spatiotemporal patterns for both the $(\alpha, \beta)=(1.5,1.5)$ and $(2,2)$ cases. Figures $7(\mathrm{a})$ and $7(\mathrm{~d})$ correspond to the same initial conditions, only the diffusion coefficients differ $((\alpha, \beta)=(1.5,1.5)$ and $(2,2)$ respectively), similarly for Figures 7(b) and 7(e) and Figures 7(c) and 7(f).

Figure 7(a) resembles Figure 2(a) in that the apparently horizontal lines are slightly U-shaped. The stripes for the anomalous case, however, do not breathe as do those in the regular diffusion 


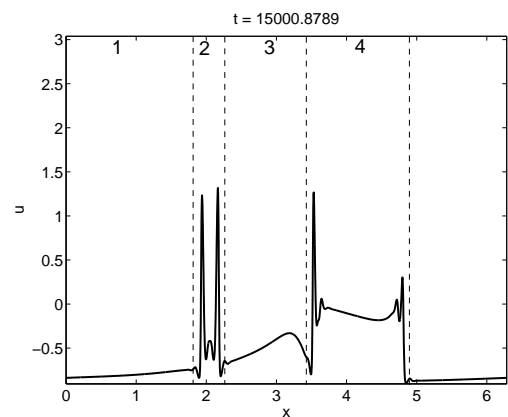

(a)

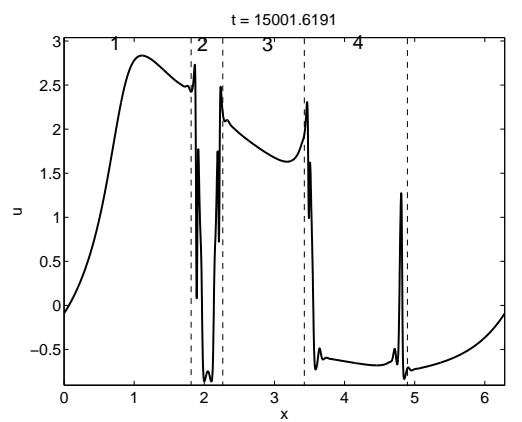

(d)

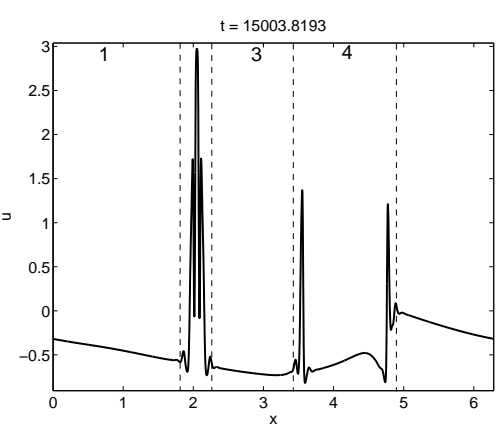

$(\mathrm{g})$

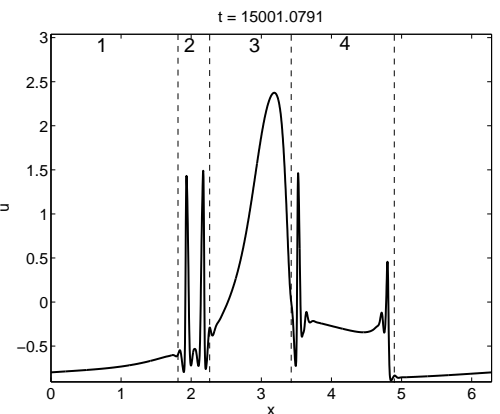

(b)

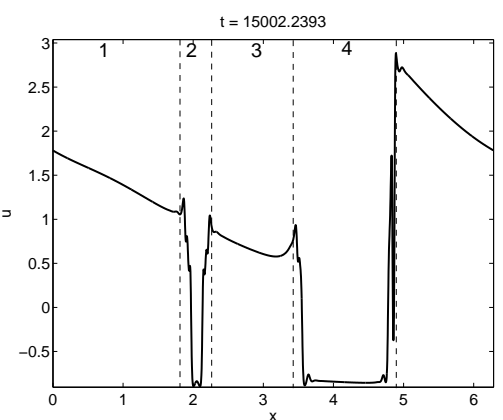

(e)

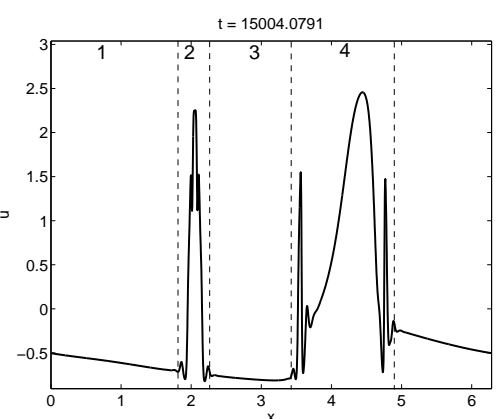

(h)

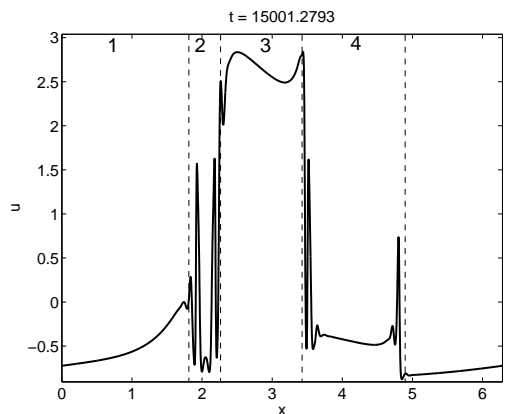

(c)

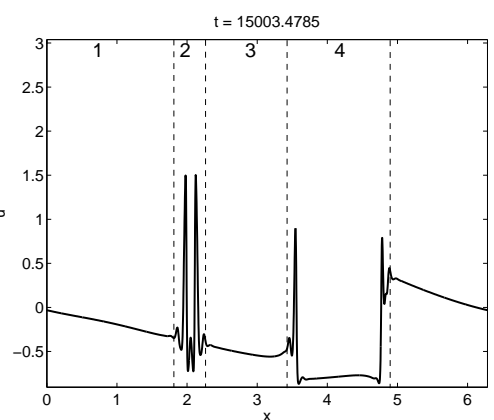

(f)

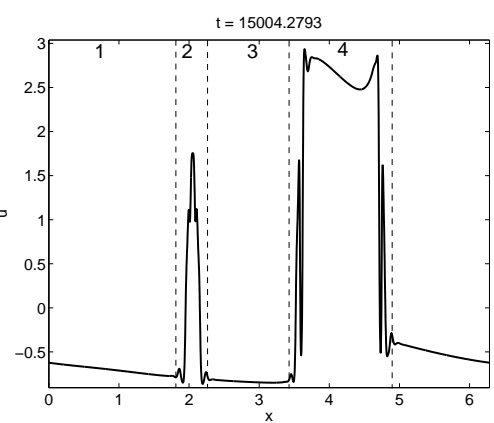

(i)

Figure 6: Plots of $u(x)$ at specific instants of time illustrating the structure of the regions in Figure 2(c). Regions 2 and 4 are near the end of a decay cycle in (a) as a spot in region 3 begins to form. The spot grows and propagates to the left ((b) and (c)) as a spot grows just to the left of $x=2$ in region 1 . The spot in region 1 grows and propagates to the left before being annihilated at $x \simeq 5$ ((d) and (e)). As regions 1 and 3 decay ((f)), spots begin to form in regions 2 and $4((\mathrm{~g})$ and (h)). The growth of region 2 occurs slightly before that of region 4 , and so it decays earlier ((i)). 


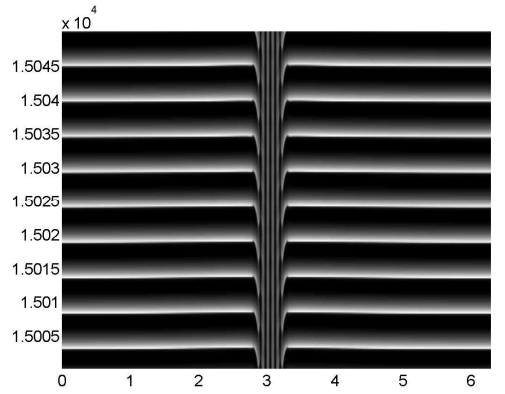

(a)

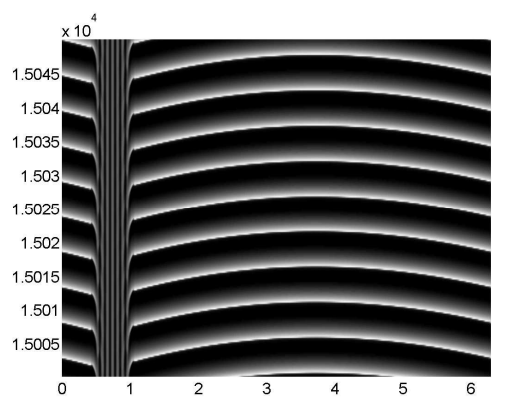

(d)

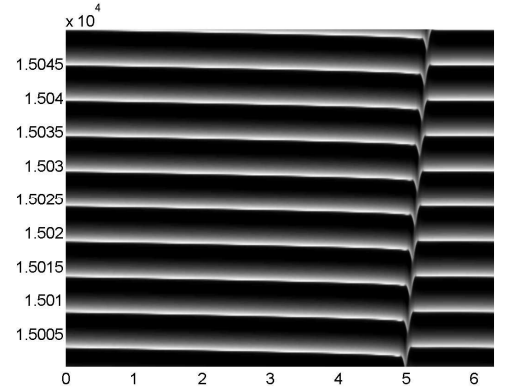

(b)

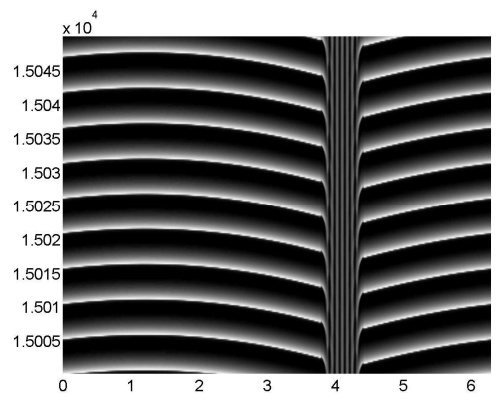

(e)

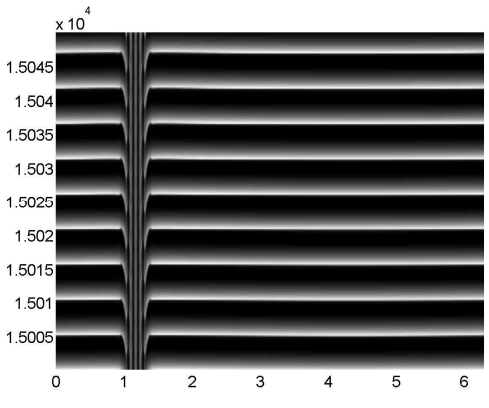

(c)

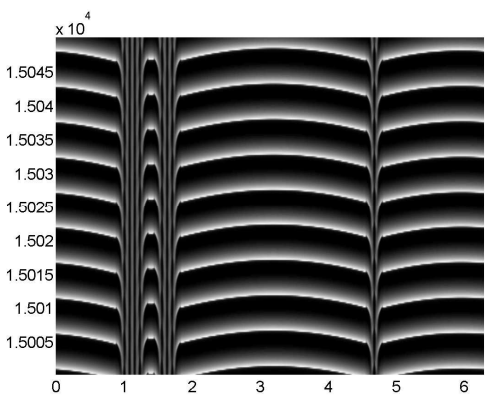

(f)

Figure 7: Spatiotemporal patterns of $u$ for $\alpha=\beta=1.5$ ((a), (b), (c)) and $\alpha=\beta=2$ ((d), (e), (f)), with $z=1.4, B=6.76$, and $\eta=0.642$. Each pair (a) and (d), (b) and (e), and (c) and (f) are generated from the same set of random initial conditions. As with Figure 2, most of the patterns for this parameter set have low spatial frequency and are mainly time-oscillatory. The main difference is in the presence of the inverted U's of the regular diffusion figures versus the flat oscillatory structures of the anomalous figures.

case. Comparing Figures 7(a) and 7(d), we see that for the regular diffusion case, instead of there being a spot created away from the stripes that splits into two spots that are annihilated on either side of the stripes (U-shape), two spots are generated on either side of the stripes, which then oppositely propagate and are annihilated between the generation sites, resulting in an inverted Ushape. In Figure 7(d), the left spot fires first, resulting in an annihilation site that is closer to the right side of the stripes than to the left. Thus, for the anomalous case, there is creation away from the stripes and annihilation at the stripes (U-shaped), while for regular diffusion, there is creation at the stripes and annihilation away from the stripes (inverted $U$ ).

Figure 7(b) (anomalous) shows a single traveling dislocation that propagates to the right. Periodically, a spot is generated on the left side of the dislocation, which then propagates until it hits the right side of the dislocation. This steady state is similar to but simpler than the pattern in Figure 
2(c). There are three periods associated with this steady state: the time it takes for a newly formed spot to travel from one side of the dislocation to the other ( $\sim 1.08$ time units), the time between two spot-creation events $(\sim 5.2092$ time units $)$, and the time it takes for the dislocation to travel one length of the system ( $\sim 939.039$ time units). None of the ratios computed from these periods appear to be a simple rational number. While all results of the computation are necessarily rational, this suggests that in reality the periods are incommensurate, a situation that can lead to chaos, but does not seem to do so in this case. Figure 7(c) is similar to Figure 7(a), the only difference being the number of stripes. Figures 7(d) and 7(e) appear to be the same solution modulo a shift in space.

In Figure 7(f), unlike in Figure 7(d), all spots appear to fire simultaneously. The small inverted U's embedded in the stripes are similar to those found in Figure 2(b). The dynamical behavior of one of the larger inverted U's centered at $x \simeq 3.2$ of Figure 7(f) is illustrated in more detail in Figures 8(a) - 8(d), where $u$ is plotted against $x$ for a restricted $x$ interval. They correspond to the inverted $\mathrm{U}$ that occurs between $t=1.5005 \times 10^{4}$ and $t=1.501 \times 10^{4}$. Figure 8(a) shows the formation of two spots A and B. They co-propagate and grow (Figure 8(b)) before meeting and annihilating in the middle while two new spots are formed in their place (Figures 8(c) and 8(d)). The new spots are labeled $\mathrm{A}^{\prime}$ and $\mathrm{B}^{\prime}$ in Figure 8(d).

Figures 9(a) - 9(c) show spatiotemporal patterns of single or multiple localized structures in a sea of stripes for $\alpha=\beta=1.5, z=1.6, B=7.76$ and $\eta=0.67$. Figure 9(d) is a close-up of Figure $9(\mathrm{c})$. Using the same initial conditions, only stationary stripes were found for the case of regular diffusion.

The predominant structures seen for this parameter set are the square-shaped spatiotemporal cells that take on the shape of an inverted $U$ with a flat apex, which we did not find for regular diffusion. It appears that, depending on the initial conditions, these cells can occur in different sizes and numbers, and can have any relative spacing between them. Some square cells behave in a similar fashion as the inverted U's of Figure 7(f) in that a small spot arises rapidly between two slowly growing and oppositely propagating spots, which are subsequently absorbed into the interior spot. However, there are some significant differences described below. For some square cells this is a spatially symmetric process that results in a concentrated bright spot at the apex. Other square cells can exhibit either symmetric or slightly asymmetric behavior. In either case, the interior spot is much wider and is less localized than that of the inverted U's in Figure 2(b), accounting for the absence of a bright spot at the apex.

Figures 10(a)-10(d) illustrate the detailed dynamics of the symmetric square cell shown in Figure 9(c) (closeup in Figure 9(d)). These figures are plotted only over a restricted $x$ interval corresponding to the extent of the square cell. An interior spot (spot A in Figures 10(a) and 10(b)) grows in the middle of two oppositely propagating spots (spots B and C in Figures 10(a) and 10(b)). Spot A then splits into two counter propagating spots (spots D and E in Figure 10(c)), which merge with spots B and C (Figure 10(c)). The solution over the entire interval then decays as two new spots form at the edge of the square cells to replace spots $\mathrm{B}$ and $\mathrm{C}$ (spots $\mathrm{B}^{\prime}$ and $\mathrm{C}^{\prime}$ in Figure 10(d)). The process then repeats periodically. The primary difference between these patterns and the inverted U's found with regular diffusion, e.g., Figure 2(b), is that the interior spot splits into two rapidly counter propagating spots over such a rapid timescale that the apex appears flat and the interval of the cell appears to "fire" as one (essentially flat) unit. 


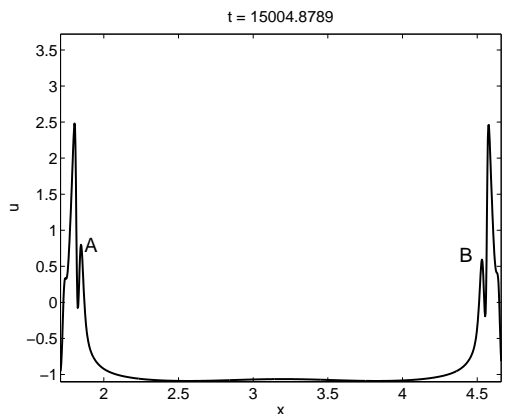

(a)

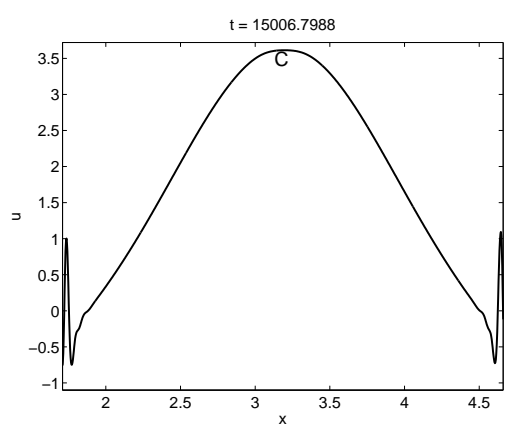

(c)

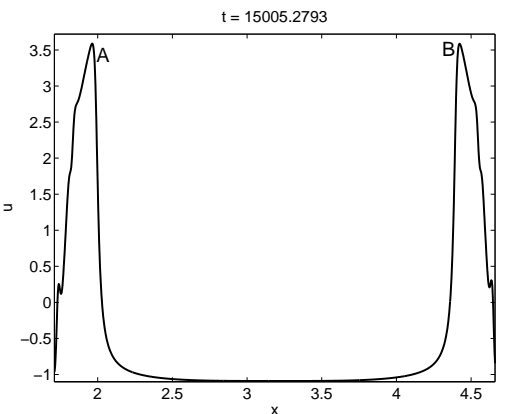

(b)

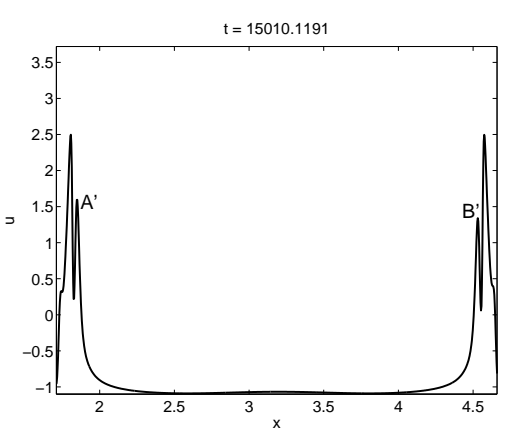

(d)

Figure 8: Line plots describing details of the spatiotemporal cell centered at $x \simeq 3.2$ in Figure 7(f). Two spots are created at the stripes ((a)), which then co-propagate and grow ((b)) before meeting and annihilating in the middle while two new spots are formed in their place ((c) and (d)).

In an asymmetric square cell, (e.g., the cell centered at $x \simeq 5.3$ in Figure 9(a)), either a spot forms closer to one of the oppositely propagating spots, or one of the oppositely propagating spots itself grows and propagates more quickly. The dynamics of this square cell exhibiting the latter scenario is illustrated in Figures 11(a)-11(d). A growing spot (spot B in Figure 11(a) and 11(b)) propagates toward the more slowly growing spot (spot A). The two spots then merge leading to an asymmetric structure (Figure 11(d)).

We next consider the effect of deterministic initial conditions. Figures 12(a) - 12(g) are generated using $u=\cos m x$ and $v=\sin m x$ for various $m$ as initial conditions. All other parameters are the same for all of the figures and are the same as the parameters employed in Figures 9(a)-9(c). For $m=1, \ldots, 10$, in cases when we found spatiotemporal cells (when $m=1,2,3,4,5,8,9$ ), aside from the two cells in the $m=1$ case (Figure 12(a)), the number of square cells was equal to $m$. The square cells were all uniformly spaced, symmetric and of the same size. For $m=6,7,10$, we found stationary stripes. For $m>10$ we have only computed for $m=60$ and $m=180$ which 


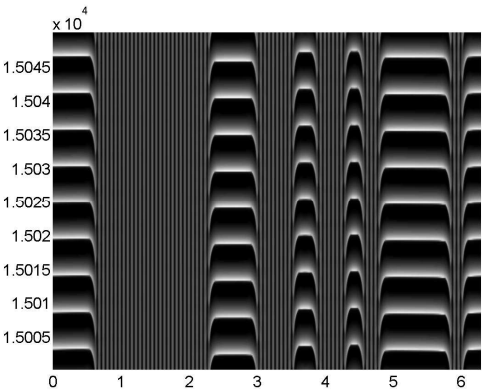

(a)

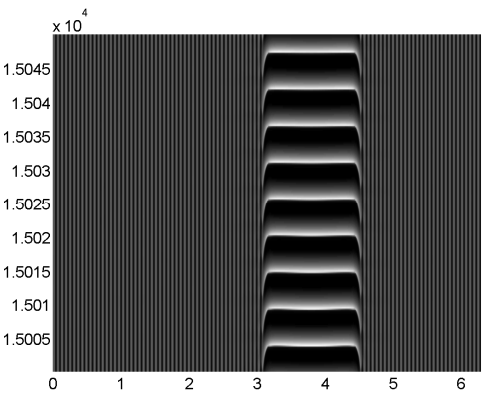

(c)

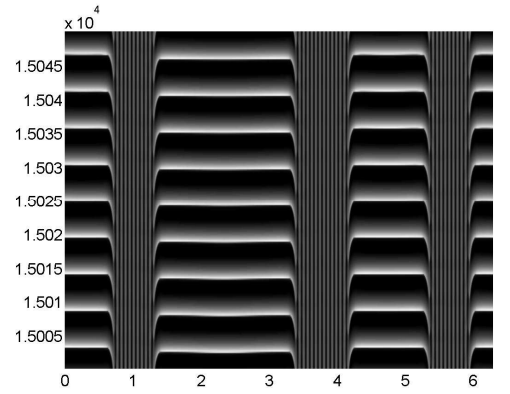

(b)

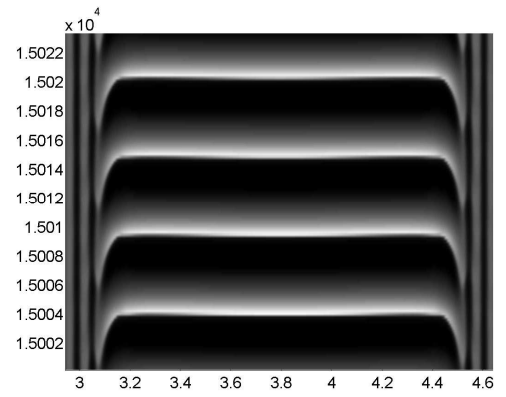

(d)

Figure 9: Spatiotemporal patterns of $u$ for anomalous diffusion with $\alpha=\beta=1.5, z=1.6$, $B=7.76$ and $\eta=0.67$. The figures differ only in initial condition. Compared to Figures 2(a)2(c) and Figures 7(a) - 7(f), these figures show a more Turing-dominant structure in which timeoscillatory structures are embedded. (d) is a close-up of the spatiotemporal cell of (c). Depending on the initial conditions, the spatiotemporal cells can occur in any size, number, and with any relative spacing.

yielded pure Hopf (horizontal stripes) and pure Turing (stationary vertical stripes), respectively.

The individual square cells are similar to the symmetric square cells obtained for random initial conditions, e.g., Figure 9(c). The primary effect of the deterministic initial conditions is that the steady state involves uniformly spaced and equal sized square cells. The effect of sinusoidal initial data for regular diffusion was similar, as in most cases we obtained cells equal in number to $\mathrm{m}$. In some cases, we obtained identical evenly spaced cells that were either symmetric or asymmetric, depending on the value of $m$. In other cases, the cells differed in size,were not evenly spaced, and differed in number from $m$. For certain values of $m$, we also obtained breathing stripes. In all cases, the apex of all cells had a marked inverted $U$ shape, in contrast to the cells with flat apexes obtained in the anomalous case.

In summary, for all three $(\alpha, \beta)$ pairs, stationary stripe patterns were observed for small $z$, 


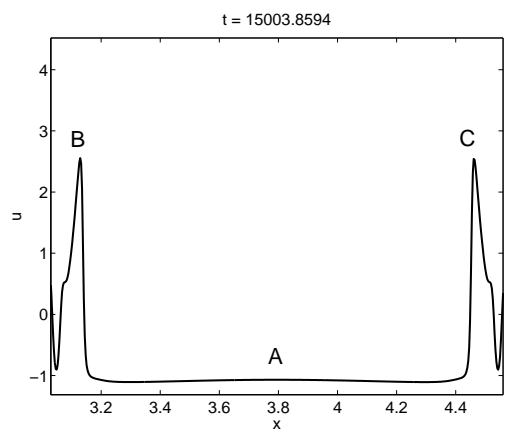

(a)

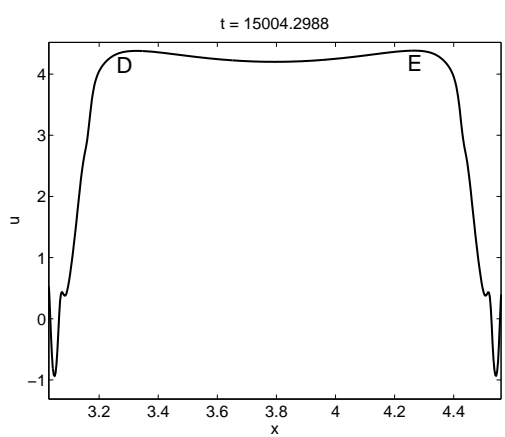

(c)

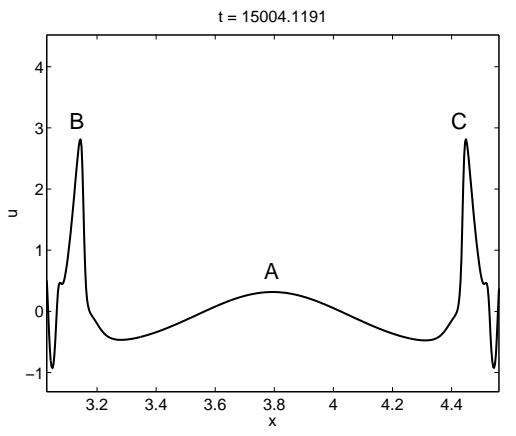

(b)

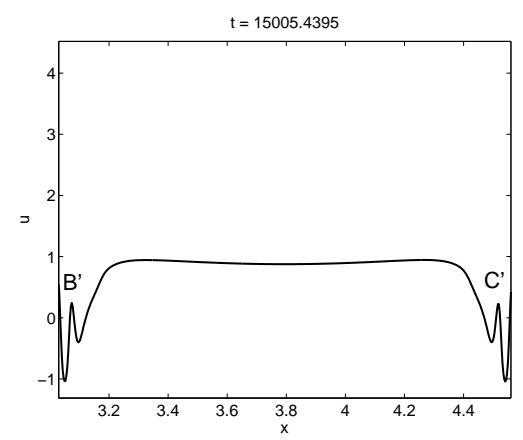

(d)

Figure 10: Line plots describing details of the symmetric spatiotemporal cell of Figure 9(d) at specific instants of time. A spot is created in between two oppositely propagating spots ((a) and (b), which splits into two counter propagating spots that are annihilated with the oppositely propagating spots (c). Two new spots are formed to replace the two oppositely propagating spots (d).

followed by mainly spatially homogeneous oscillations (horizontal stripes) for larger $z$. In the case of $(\alpha, \beta)=(1.1,1.1)$, the steady states returned to stationary stripes as $z$ was increased. In the case of $(\alpha, \beta)=(1.5,1.5)$ and $(2,2)$, steady states with spatiotemporal patterns were observed as $z$ was increased before the steady states returned to mainly stationary stripes.

Spatiotemporal patterns were observed with $(\alpha, \beta)=(2,2)$ for $z=1.2$ and 1.4 , while for $(\alpha, \beta)=(1.5,1.5)$, they were observed for $z=1.4,1.6$, and 1.8 (the $z=1.8$ case yielded nothing that had not been seen with $z=1.4$ and $z=1.6$ and is not shown). For both regular and anomalous diffusion there seem to be a large number of stable steady states, as in virtually all cases different initial conditions gave different steady states. In both cases, as $z$ was increased, the steady states became more stripe-dominated, while for the same $z$, regular diffusion yielded more stripe-dominated steady states.

In general, patterns obtained with regular diffusion were more diverse, as breathing stripes 


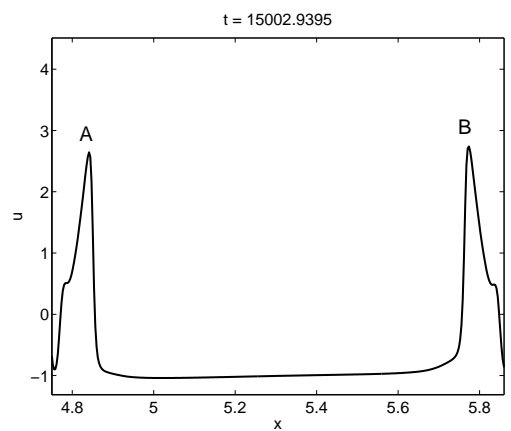

(a)

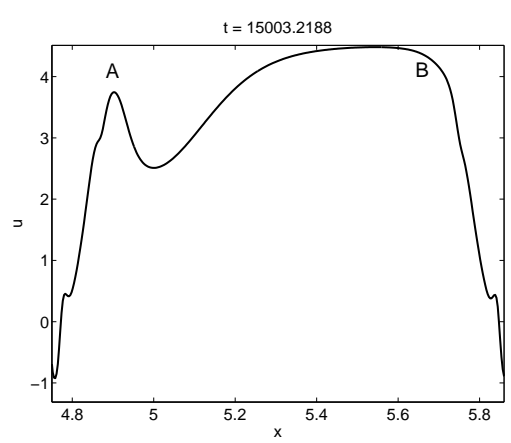

(c)

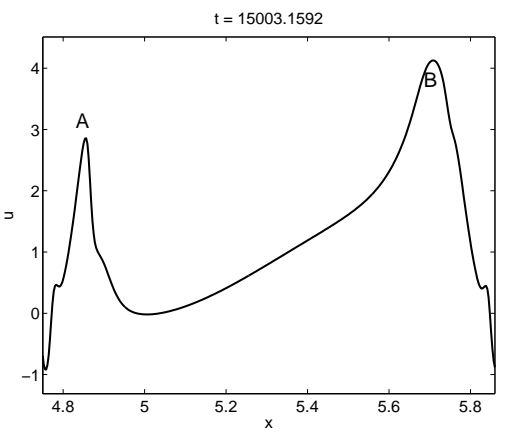

(b)

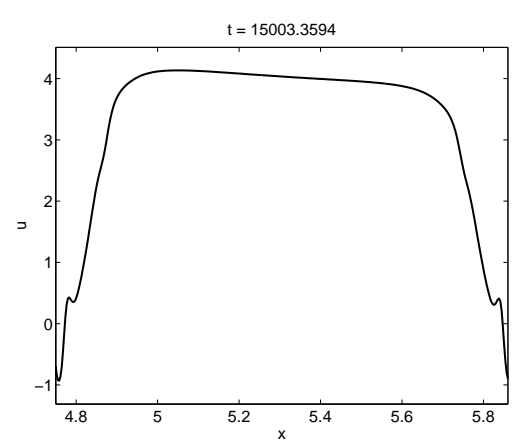

(d)

Figure 11: Line plots describing details of an asymmetric spatiotemporal cell of Figure 9(a) centered at $x \simeq 5.3$ at specific instants of time. Spot B grows and propagates more quickly than spot A ((b) and (c)) so that the spots merge at a location closer to spot A ((d)).

(Figure 2(a)), inverted U's with a pronounced peak (Figure 2(b)), multiple traveling dislocations (Figure 2(c)), and inverted U's (Figure 7(d)) were not observed for anomalous diffusion. For anomalous diffusion, the predominant pattern appears to be that of spatiotemporal cells in the shape of inverted U's with a square apex, embedded in a mainly Turing structure of vertical stripes. This is something that we did not find with regular diffusion. With random initial data, the cells occur in different sizes and numbers, and stable steady states with essentially any inter-cell spacing and number of cells appear possible.

For sinusoidal initial conditions with wave number $m$ only steady states with symmetric and uniformly spaced cells (with flat apexes) were found. With $m=O(1)$ the resulting steady state had exactly $m$ cells with the exceptions described above. For larger values of $m$, only pure Turing and pure Hopf steady states were found. In the case of regular diffusion, breaks in the symmetry of the steady states were observed, as described above, and the number of cells was not always equal to $m$. 


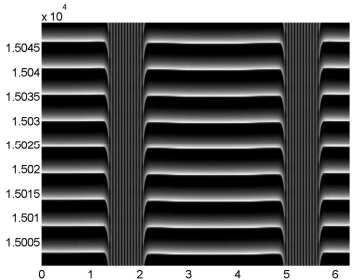

(a)

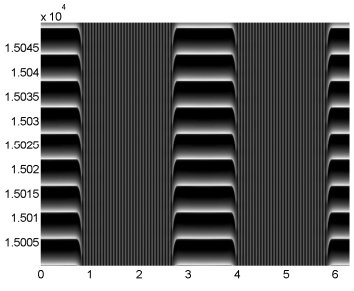

(b)

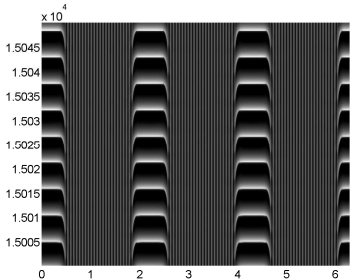

(c)

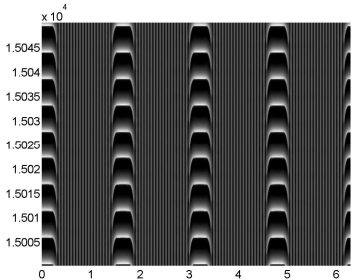

(d)

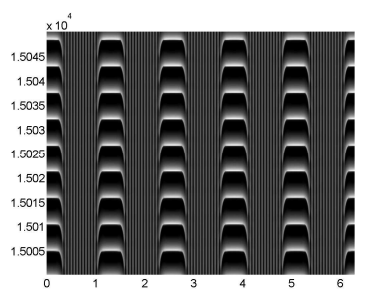

(e)

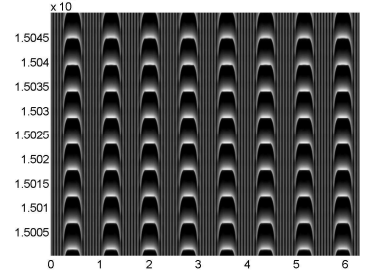

(f)

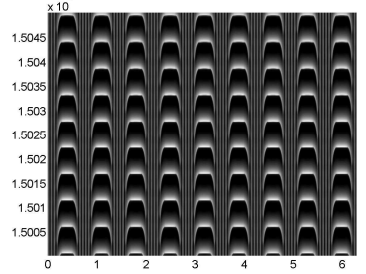

(g)

Figure 12: Spatiotemporal patterns of $u$ for anomalous diffusion with $\alpha=\beta=1.5, z=1.6$, $B=7.76$ and $\eta=0.67$. Initial conditions were $u=\cos m x, v=\sin m x$ with (a) $m=1$, (b) $m=2$, (c) $m=3$, (d) $m=4$, (e) $m=5$, (f) $m=8$, and (g) $m=9$. With the exception of (a), the number of spatiotemporal cells is equal to $m$ and all cells in each figure are of the same size and spaced evenly, and also oscillate in phase. For $m=1$, the number of cells is $2 m=2$, and while the cells are spaced evenly, they are not equal in size and oscillate out of phase.

\subsection{The fully nonlinear regime with unequal diffusion exponents}

We next consider the case $\alpha \neq \beta$. In this case $s$ is no longer equal to 1 so that for the same value of $z$ the values of $B_{c r}$ and $\eta_{c}$ are slightly different from the $\alpha=\beta$ case. Since we do not change the values of $\mu$ and $\eta_{2}$, both $B$ and $\eta$ change as a result of the unequal diffusion exponents. To keep these parameters from changing would require altering how far the system is into the nonlinear regime as well as its closeness to the C2THP, and this could result in a significant qualitative change in the patterns not attributable to unequal diffusion exponents.

We first kept $\beta=1.5$ while setting $\alpha$ to 1.4 and then to 1.6. For each $\alpha$, we computed steady states starting from three sets of initial conditions: the same random initial conditions used in Section 5.1, the same sinusoidal initial conditions used in Section 5.1, and the steady states computed from random initial conditions with $\alpha=\beta=1.5$ and $z=1.6$. In this last case, we took the solutions $u(x)$ and $v(x)$ at $t=1.5 \times 10^{4}$ and set them as initial conditions for computations with the same $z$ and different $\alpha$.

For $\alpha=1.6, \beta=1.5$, and $z=1.6(B=8.57, \eta=0.672)$, the only steady states we found were stationary stripes. In contrast, for $\alpha=1.4, \beta=1.5$ we did find spatiotemporal patterns. 
Figures 13(a) - 13(c) show steady states for this case obtained from the same initial conditions employed in Figures 9(a) - 9(c). Figure 13(d) is a closeup of the solution shown in Figure 13(c).

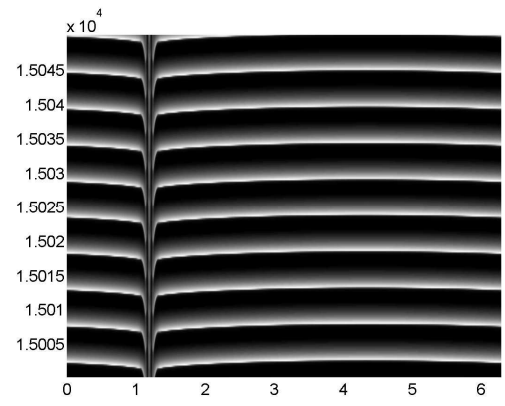

(a)

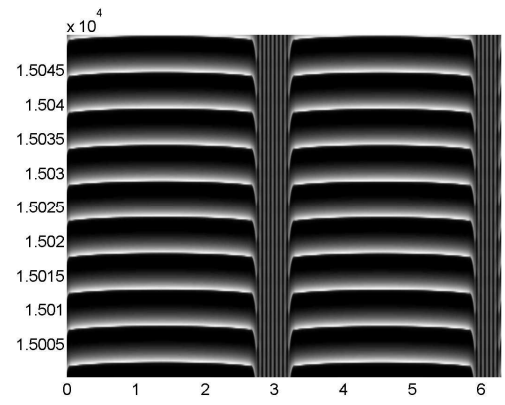

(c)

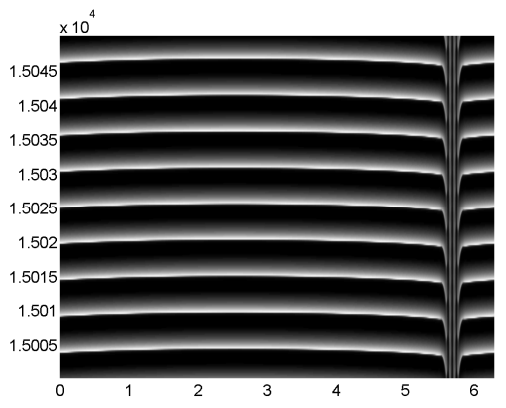

(b)

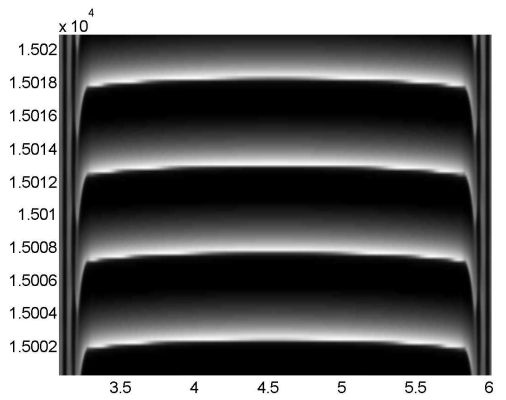

(d)

Figure 13: Spatiotemporal patterns of $u$ for anomalous diffusion with $\alpha=1.4, \beta=1.5, z=1.6$, $B=7.11$ and $\eta=0.66$. The figures differ only in initial condition. The same random initial conditions were used in Figures 9(a) - 9(c), and match up according to letter label. In comparison to Figures 9(a) - 9(c), these show a more Hopf-dominant set of steady states. Figure (d) is a close up of the right spatiotemporal cell of (c). The cells in (a) - (d) also exhibit a noticeable inverted U shape, compared to those of Figures 9(a) - 9(d), which exhibit a very slight U-shape. (a) and (b) are similar but differ in the number of stripes.

There are two primary differences between Figures 13(a) - 13(c) (unequal diffusion exponents) and Figures 9(a) - 9(c) (equal diffusion exponents). In the former case regions of vertical stripes (Turing type modes) are very much reduced compared to the equal diffusion exponent case. For the unequal diffusion case, the solution appears to be dominated by nearly spatially homogeneous regions that oscillate in time (i.e., Hopf type regions). The second difference is that in contrast to the very slight $\mathrm{U}$-shaped apexes for the $\alpha=\beta$ case solutions with $(\alpha, \beta)=(1.4,1.5)$ have a very marked inverted U-shaped apex, similar to those seen in Figures 7(d) - 7(f) for $(\alpha, \beta)=(2,2)$.

A similar difference was observed when the initial conditions were set as sinusoidal functions. 
Figures 14(a) - 14(j) show more Hopf-dominated steady states than do Figures 12(a) - 12(g), which correspond to steady states with $\alpha=\beta=1.5$. Further, unlike the $\alpha=\beta=1.5$ case, spatiotemporal patterns were obtained for sinusoidal initial conditions of all wave numbers $m=1, \ldots, 10$.

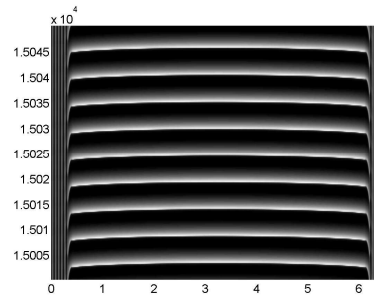

(a)

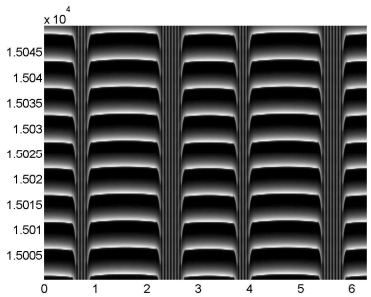

(b)

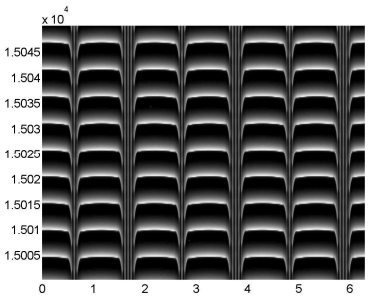

(c)

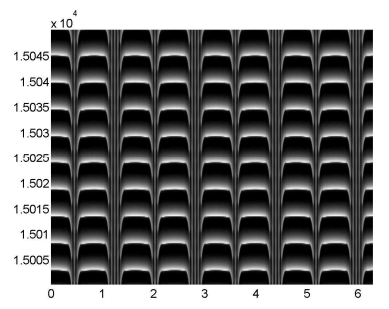

(d)

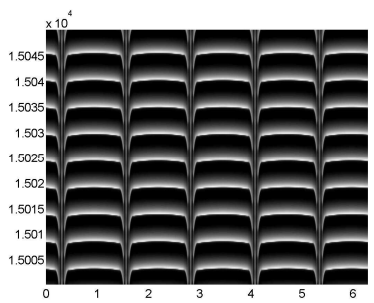

(e)

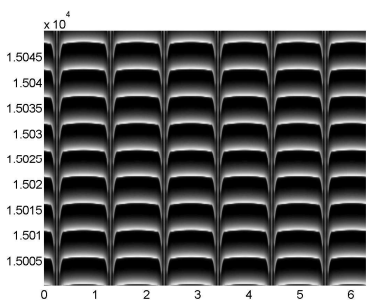

(f)

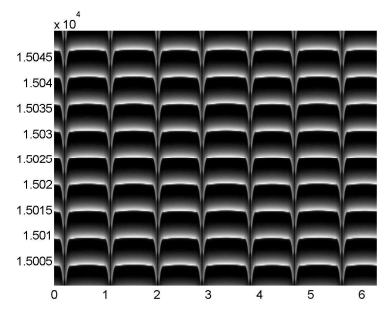

(g)

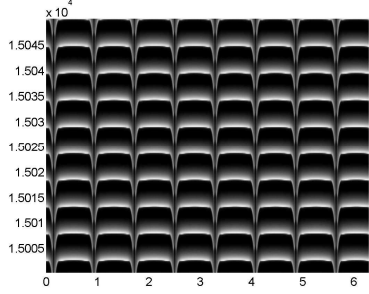

(h)

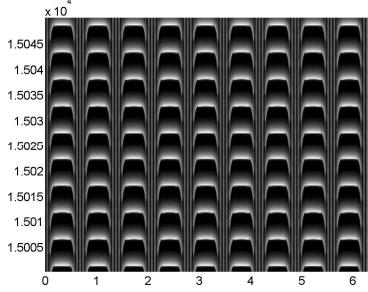

(i)

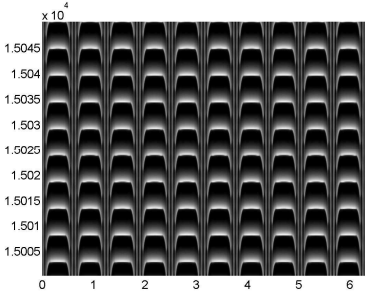

(j)

Figure 14: Spatiotemporal patterns of $u$ for anomalous diffusion with $\alpha=1.4, \beta=1.5, z=1.6$, $B=7.11$ and $\eta=0.66$. Initial conditions were $u=\cos m x, v=\sin m x$ with (a) $m=1$, (b) $m=2$, (c) $m=3$, (d) $m=4$, (e) $m=5$, (f) $m=6$, (g) $m=7$, (h) $m=8$, (i) $m=9$, and (j) $m=10$. Unlike in Figure 12(a), the $m=1$ case ((a)) contains only one spatiotemporal cell. (b) (d) contain pairs of cells equal in number to $m$. In (b), the two smaller cells oscillate in phase with each other, as do the two larger cells. All cells in all other figures are of equal size and oscillate in phase. In (e) - (j), the trend is the same as in the case of equal diffusion exponents in that the number of cells is equal to $m$.

As in the case with equal diffusion exponents, the $m=1$ case (Figure 14(a)) appears to be an 
exception. For $m=2,3,4$ (Figures 14(b) - 14(d)), we see $m$ pairs of cells so that the total number of cells is $2 m$. This is in contrast to the $m=1$ case for which there is only one square cell. This is also in contrast to the case of equal diffusion exponents, where generally the number of square cells was $m$, not $2 m$. This suggests that the cells split as $\beta-\alpha$ increases from zero. The $m=2$ case is also an exception in that there are cells of unequal size that oscillate with a phase difference. In all other cases, the cells are of equal size and oscillate in phase. In Figures 14(e) - 14(j) we do not find cell splitting and the number of cells equals $m$, similar to the $\alpha=\beta$ case.

When we used the steady state solutions found in Figures 9(a) - 9(c) as initial conditions for the $(\alpha, \beta)=(1.4,1.5)$ runs, there were cases when the steady state became more Turing dominated and the spatiotemporal cells attain a more inverted U shape (e.g. Figure 15(a)) and cases when the difference was only in the shape of the cells (e.g. Figures 15(b) and 15(c)). Note that Figure 15(a) is a continuation of Figure 9(a) with a different value for $\alpha$, Figure 15(b) of Figure 9(b), and Figure 15(c) of Figure 9(c).

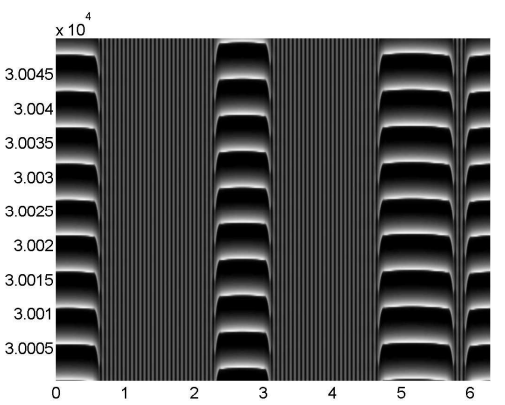

(a)

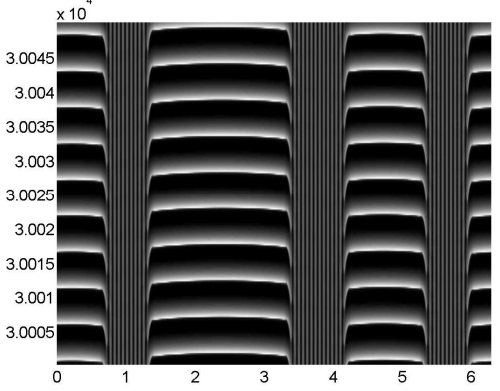

(b)

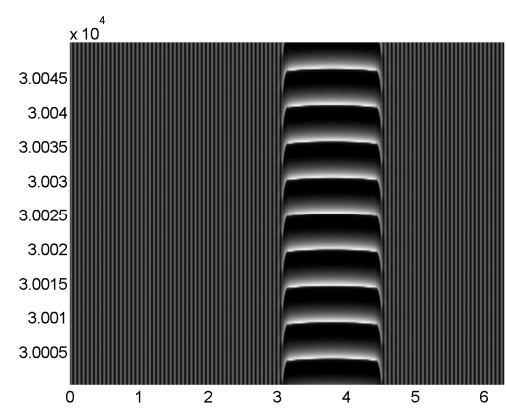

(c)

Figure 15: Steady states with $\alpha=1.4, \beta=1.5$ using as initial conditions the steady states computed with $\alpha=\beta=1.5$ (Figures 9(a) - 9(c), respectively). The only difference between (b) and 9(b) and (c) and 9(c) is that the cells of (b) and (c) have a more rounded apex. Going from Figure 9(a) to (a), we see that, in addition to the more rounded cells, the number of cells has decreased, yielding a more Turing-dominant solution.

For the $(\alpha, \beta)=(1.6,1.5)$ case, while we obtained only pure Turing steady states with $z=1.6$, we found spatiotemporal patterns with $z=1.4(B=7.35$ and $\eta=0.649)$ when starting with the same random initial conditions used throughout. Compared to the $z=1.4$ runs with equal diffusion exponents, Figures 16(a) - 16(c) show more Turing-dominant steady states.

The predominant feature in Figures 16(a) - 16(c) is the presence of spatiotemporal cells with a U-shaped apex, which are described in Section 5.1. They indicate that a spot, born away from the stripes, splits into two counter propagating spots, which are then annihilated at the stripes. The difference between the cells of Figures 16(a) - 16(c) and those described in Section 5.1 is that, in the former, the two spots on either side of the cell do not grow as large as they do in the latter, which is why the U-shape is much more noticeable in the former. The two smaller cells in Figure 


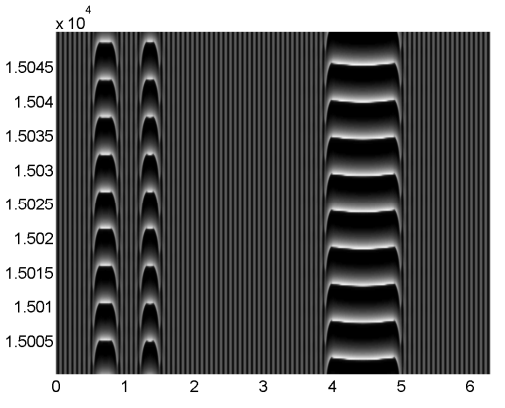

(a)

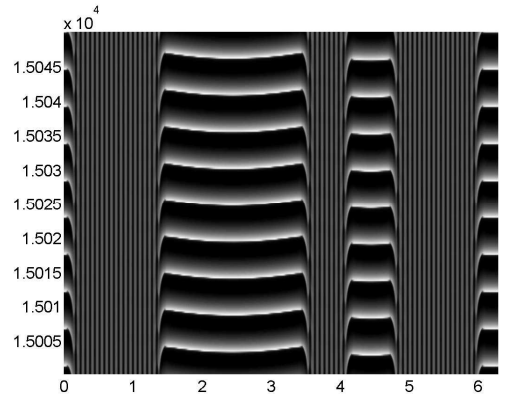

(b)

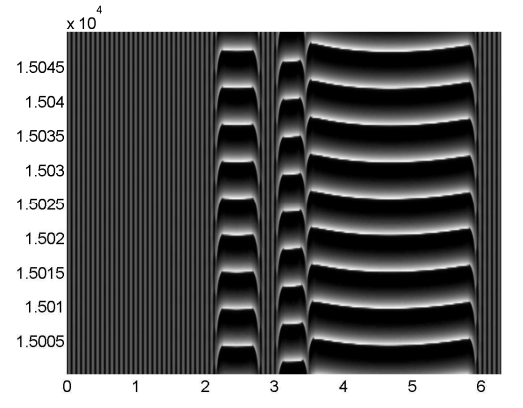

(c)

Figure 16: Spatiotemporal patterns of $u$ for anomalous diffusion with $\alpha=1.6, \beta=1.5, z=1.4$, $B=7.35$ and $\eta=0.649$. The figures differ only in initial condition. The two smaller cells of (a) resemble the inverted U's of Figure 7(f). The cells in (b) are all qualitatively similar, while there are two immediately adjacent asymmetric cells in (c).

16(a) are similar to the small inverted U's of Figure 7(f) and oscillate in phase. The cells of Figure 16(b) are qualitatively similar, while in Figure 16(c), we see an asymmetric spatiotemporal cell immediately adjacent to a U-shaped cell, which is also asymmetric in that the left spot decays slightly more slowly than does the right.

When we set one of the diffusion exponents equal to 2 (regular diffusion) and the other to a value less than 2 (anomalous diffusion), most of the patterns that we found were comparable to those described above. However, for $(\alpha, \beta)=(2,1.4)$, and $z=1.4(B=87.4, \eta=0.708)$, starting with random initial conditions, we found a steady state consisting entirely of spatiotemporal spots (Figures 17(a) and 17(b)). Different random initial conditions yielded qualitatively very similar steady states, the only difference being the size of the individual spots. While the pattern of spots may be due in part to the much larger value of $B$, from the limited number of computations that we performed, we did not find any similar steady states for the same or comparable values of $B$ under regular diffusion. Note a similar spot pattern was found by De Wit et al. for regular diffusion in [7] with $B=10.3$, closer to the weakly nonlinear regime and farther from the C2THP.

The dynamics of the spots of Figures 17(a) and 17(b) is illustrated in Figures 18(a) - 18(d), which depict half of a period (approximately 2 units of time) and correspond to the lower half of Figure 17(b). The dark spots in Figures 17(a) and 17(b) are due to the global minima of $u$ in Figures 18(a) - 18(d), while the lightest regions are due to the traveling pulse that appears to be traveling at a constant or near constant speed, seen centered at $x \simeq 4.8$ in Figure 18(a), $x \simeq 0.6$ in Figure 18(b), $x \simeq 2.7$ in Figure 18(c) and back to $x \simeq 4.8$ in Figure 18(d). The valleys accounting for the black spots change in width, which can be seen in Figures 17(a) and 17(b), as a spot is very narrow near the beginning and end of its duration, and wider during the middle. Locations at which valleys exist turn into locations of peaks when the pulse arrives. Once the pulse passes, the valleys reappear, but with a shift in space relative to their previous locations, which can be clearly seen in 


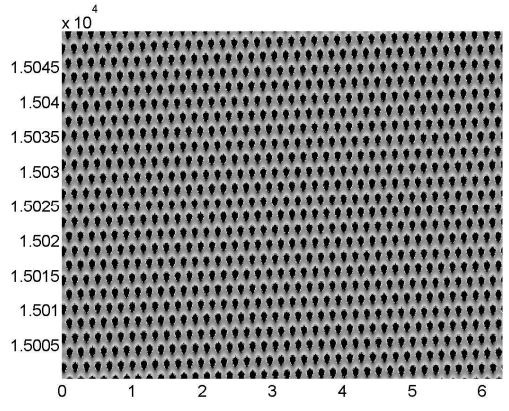

(a)

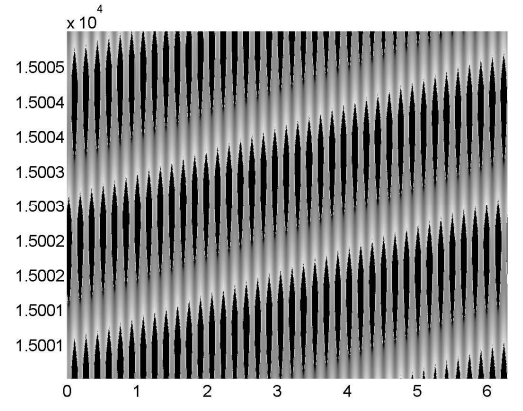

(b)

Figure 17: Spatiotemporal patterns of $u$ for anomalous diffusion with $(\alpha, \beta)=(2,1.4), z=1.4$, $B=87.4$, and $\eta=0.708$. (b) shows a closeup of the bottom portion of (a).

Figure 17(a). The result in the space-time plot is that one row of spots runs diagonally, and a row is displaced by one spot's width relative to a neighboring row. Over one full period (approximately 4 units of time), the valleys return to the same locations.

In summary, it appeared that the $(\alpha, \beta)=(1.4,1.5)$ steady states with $z=1.6$ were more Hopf-dominant than the cases with $(\alpha, \beta)=(1.5,1.5)$. In the case of the continuation runs, however, this trend was not seen. In the case of $(\alpha, \beta)=(1.6,1.5)$ with $z=1.4$, the steady states appeared to be more Turing-dominant than the same runs with $(\alpha, \beta)=(1.5,1.5)$. This trend seems more consistent, as pure Turing steady states were found for $z=1.6$ for all initial conditions mentioned above. Further, the spatiotemporal cells, which appear to have a flat apex in the $(\alpha, \beta)=(1.5,1.5)$ case, take on an inverted $U$ shape in the $(\alpha, \beta)=(1.4,1.5)$ case and a U-shape in the $(\alpha, \beta)=(1.6,1.5)$ case. In the case when one component undergoes regular diffusion and the other anomalous, we found a series of adjacent valleys that periodically turn into peaks when a traveling pulse arrives, accounting for the dark spots and their arrangement in Figure 17(a). Similar structures were found for regular diffusion in [7].

Since in all of these cases, $B$ and $\eta$ change as described above it is difficult to infer whether these differences are due solely to the difference in diffusion exponents.

\section{Conclusions}

Using weakly nonlinear analysis, we derived a pair of coupled amplitude equations that describe the evolution of the Turing and Hopf modes over a long time scale near a C2THP of the superdiffusive Brusselator model. The amplitude equations have a similar form to those of the regular model, but differ in three regards: the two spatial derivatives in the pair of equations are with respect to two different length scales, the equation describing the evolution of the Hopf mode contains an integro-differential operator, which reflects the non-local effect of anomalous diffusion, 
and finally, the coefficients of the amplitude equations differ from those of the regular amplitude equations. The latter two differences contribute to the differences in the long wave stability criteria of the special solutions, which are described in Section 4. Two of these criteria were confirmed with numerical computations in the weakly nonlinear regime, while criteria involving both the Turing and Hopf modes were inconclusive due to numerical difficulties arising from small $\epsilon$.

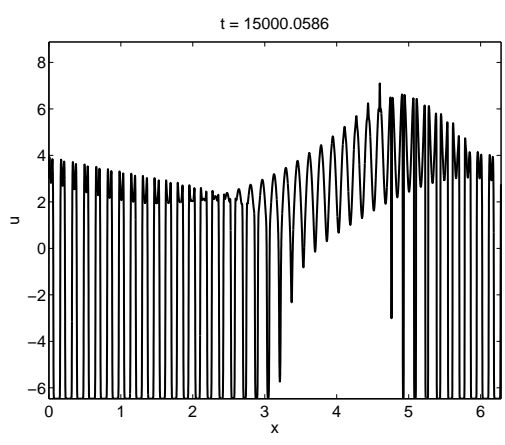

(a)

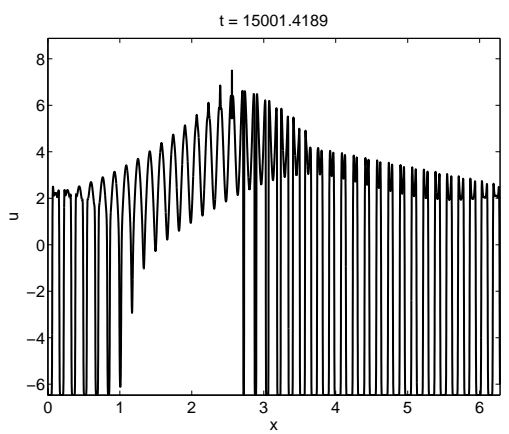

(c)

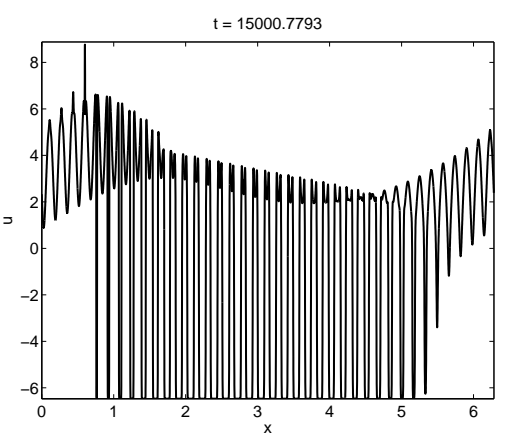

(b)

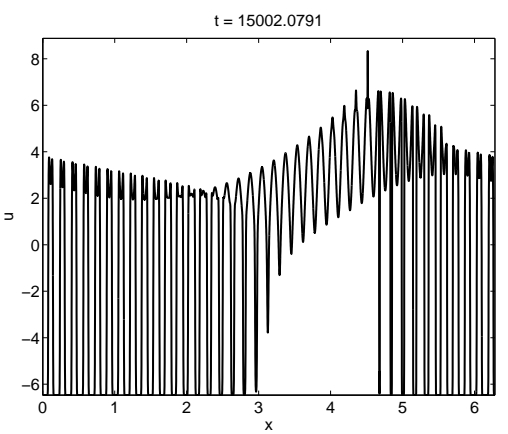

(d)

Figure 18: Line plots describing details of the spots of Figures 17(a) and 17(b) at specific instants of time. The global minima, account for the dark spots in Figures 17(a) and 17(b).

In computations in the fully nonlinear regime, one of the dominant spatiotemporal structures that we found under anomalous diffusion were the square-shaped spatiotemporal cells with either a flat apex $(\alpha=\beta)$, an inverted $\mathrm{U}$ shaped apex $(\alpha<\beta)$, or a $\mathrm{U}$-shaped apex $(\alpha>\beta)$. Qualitatively similar structures were found under regular diffusion with a similar range in values of $z$, though the square and flat cells embedded in a mainly Turing structure was something that we did not find under regular diffusion. There was also behavior that we found under regular diffusion that we did not find with anomalous diffusion, such as breathing stripes and multiple traveling dislocations that appear to repel each other. With equal diffusion exponents, it appeared that the effect of anomalous diffusion was to delay the onset of Hopf-type behavior in terms of increasing $z$, and 
also to inhibit spatiotemporal pattern formation, as was seen when we found only pure Turing and pure Hopf solutions with $\alpha=\beta=1$.1. The main effect of unequal but close diffusion exponents appeared to be to alter the apex shape of the spatiotemporal cells from the case of equal diffusion exponents. When one diffusion was regular and the other anomalous, we found spatiotemporal spots corresponding to valleys whose sizes and positions changed periodically, similar to structures presented in [7]. More generally, we found a large number of steady state patterns consisting of a localized region or regions of stationary stripes in a background of time periodic cellular motion, as well as a localized region or regions of time periodic cells in a background of stationary stripes. Each such pattern lies on a branch of such solutions, is stable and corresponds to a different initial condition. The patterns correspond to the phenomenon of pinning of the front between the stripes and the time periodic cellular structure. The different branches live in a region called the pinning region in which such solution branches snake back and forth. The idea of pinning was originally suggested by Pomeau [13], who referred to pinning as locking. The idea has been considered and extended by a number of researchers, including Knobloch and coauthors [1, 3, 4], who considered localized stationary patterns in a background of a stationary, spatially uniform state, as well as in a background of small amplitude traveling waves, Bensimon et. al. [2], who considered localized traveling rolls (stripes) in a background of a stationary, spatially uniform state, and Malomed et. al [9], who considered localized hexagonal patterns in a background of stationary stripes. These scenarios involve a subcritical bifurcation leading to bistability between the basic state and the bifurcated state after the latter turns around to become stable. In contrast, our study involves bistability between two stable supercritical branches which exist near a codimension two bifurcation point. We note that many of these patterns disappear when the size $L$ of the domain is considerably reduced.

\section{Acknowledgments}

The authors gratefully acknowledge the support of U.S. National Science Foundation grant DMS 0707445 and Natural Sciences and Engineering Research Council of Canada grant PGS D - 358595 - 2009.

\section{References}

[1] P. Assemat, A. Bergeon, E. Knobloch. Spatially localized states in Marangoni convection in binary mixtures. Fluid Dynamics Research 40 (2008), 852-876.

[2] D. Bensimon, B.I. Shraiman, V. Croquette. Nonadiabatic effects in convection. Phys. Rev. A38 (1988), 5461-5464.

[3] J. Burke, E. Knobloch. Localized states in the generalized Swift-Hohenberg equation. Phys. Rev. E73 (2006), art. 56211. 
[4] J. Burke, E. Knobloch. Homoclinic snaking: structure and stability Chaos 17 (2007), art. 037102.

[5] E.J. Crampin, E.A. Gaffney, P.K. Maini. Reaction and diffusion on growing domains: Scenarios for robust pattern formation. Bull. Math. Biol., 61 (1999), 1093-1120.

[6] A. De Wit. Spatial patterns and spatiotemporal dynamics in chemical systems. Adv. Chem. Phys., 109 (1999), 435-513.

[7] A. De Wit, D. Lima, G. Dewel, P. Borckmans. Spatiotemporal dynamics near a codimensiontwo point. Phys. Rev. E54 (1996), 261-271.

[8] A.A. Golovin, B.J. Matkowsky, V.A. Volpert. Turing pattern formation in the Brusselator model with superdiffusion. SIAM J. Appl. Math., 69 (2008), 251-272.

[9] B.A. Malomed, A.A. Nepomnyashchy, M.I. Tribelsky. Domain boundaries in convection patterns. Phys. Rev. A42 (1990), 7244-7263.

[10] R. Metzler, J. Klafter. The random walk's guide to anomalous diffusion: a fractional dynamics approach. Phys. Rep. 339 (2000), No. 1, 1-77.

[11] Y. Nec, A.A. Nepomnyashchy, A.A. Golovin. Oscillatory instability in super-diffusive reaction-diffusion systems: Fractional amplitude and phase diffusion equations. EPL, 82 (2008), 58003 (6pp).

[12] K.B. Oldham, J. Spanier. The fractional calculus. Academic Press, New York, 1974.

[13] Y. Pomeau. Front motion, metastability and subcritical bifurcations in hydrodynamics. Physica D23 (1986), 3-11.

[14] J.C. Tzou, B.J. Matkowsky, V.A. Volpert. Interaction of Turing and Hopf modes in the superdiffusive Brusselator model. Appl. Math. Lett. 22 (2009), 1432-1437.

[15] L. Yan, M. Dolnik, A.M. Zhabotinsky, I.R. Epstein. Pattern formation arising from interaction between Turing and wave instabilities. J. Chem. Phys., 117 (2002), 7259-7265. 